\title{
Higher Mahler measure for cyclotomic polynomials and Lehmer's question
}

\author{
Matilde Lalín • Kaneenika Sinha
}

\begin{abstract}
The $k$-higher Mahler measure of a nonzero polynomial $P$ is the integral of $\log ^{k}|P|$ on the unit circle. In this note, we consider Lehmer's question (which is a long-standing open problem for $k=1$ ) for $k>1$ and find some interesting formulae for 2- and 3-higher Mahler measure of cyclotomic polynomials.
\end{abstract}

Keywords Higher Mahler measures · Lehmer's question · Cyclotomic polynomials $\cdot$ Zeta values

Mathematics Subject Classification (2000) 11R06 · 11R09 - 11C08 · 11 Y35

M.L. was supported by NSERC Discovery Grant 355412-2008 and a Faculty of Science Startup grant from the University of Alberta. K.S. was supported by a Pacific Institute for the Mathematical Sciences Postdoctoral Fellowship and the above grants.

M. Lalín

Département de mathématiques et de statistique,

Université de Montréal,

Montréal, QC, H3C3J7, Canada

Tel.: 1-514-343-6689

Fax: 1-514-343-5700

E-mail: mlalin@dms.umontreal.ca

K. Sinha

Department of Mathematical Sciences,

Indian Institute of Science Education and Research Kolkata,

Mohanpur, Nadia, 741252, West Bengal, India

Tel.: 91-33-2587-3223

Fax: 91-33-2587-3019

E-mail: kaneenika@iiserkol.ac.in 


\section{Introduction}

Definition 1 Given a non-zero polynomial $P(x) \in \mathbb{C}[x]$ and a positive integer $k$, the $k$-higher Mahler measure of $P$ is defined by

$$
m_{k}(P):=\frac{1}{2 \pi i} \int_{|x|=1} \log ^{k}|P(x)| \frac{d x}{x},
$$

or, equivalently, by

$$
m_{k}(P):=\int_{0}^{1} \log ^{k}\left|P\left(e^{2 \pi i \theta}\right)\right| d \theta .
$$

We observe that for $k=1, m_{1}(P)$ is the classical (logarithmic) Mahler measure given by

$$
m(P):=\log |a|+\sum_{j=1}^{n} \log ^{+}\left|r_{j}\right|, \text { for } P(x)=a \prod_{i=1}^{n}\left(x-r_{j}\right)
$$

where $\log ^{+} t=\log \max \{1, t\}$ for a non-negative real number $t$. This object first appeared in a 1933 paper by Lehmer [9] in connection with a method for constructing large prime numbers. A generalization to multivariable polynomials appeared in a work by Mahler 10 (who was interested in tools for transcendence theory) about 30 years later. The generalization to higher Mahler measures was recently considered in [6] for the first time.

Higher Mahler measures of polynomials are usually very hard to compute, even for simple linear polynomials in one variable. However, the investigation carried out in [6] reveals direct connections between these measures and special values of zeta functions and polylogarithms. In the case of the classical Mahler measure, analogous relations with special values of $L$-functions have been explained by Deninger 4 and others in terms of evaluations of regulators in the context of Beilinson's conjectures. One of the motivations for considering higher Mahler measures (in addition to classical Mahler measures) is that they yield different periods from the ones that we obtain from the usual Mahler measure thus revealing a more complicated structure for the regulator (see 7] for more details).

One of the tools for studying general $k$-higher Mahler measures is the following:

Definition 2 For a finite collection of non-zero polynomials $P_{1}, \ldots, P_{l} \in \mathbb{C}[x]$, their multiple Mahler measure is defined by

$$
m\left(P_{1}, \ldots, P_{l}\right):=\frac{1}{2 \pi i} \int_{|x|=1} \log \left|P_{1}(x)\right| \ldots \log \left|P_{l}(x)\right| \frac{d x}{x} .
$$

Our main interest in this note is the case of $P(x) \in \mathbb{Z}[x]$ but we consider other cases as well, such as products of cyclotomic polynomials. We recall the following well-known theorem of Kronecker [5]: 
Theorem 3 Let $P(x)=\prod_{j=1}^{n}\left(x-r_{j}\right) \in \mathbb{Z}[x]$. If $\left|r_{j}\right| \leq 1$ for each $j$, then the $r_{j}$ 's are zero or roots of unity.

An immediate consequence of Kronecker's theorem is that for a non-zero polynomial $P(x) \in \mathbb{Z}[x], m(P)=0$ if and only if $P$ is monic and is a product of powers of $x$ and cyclotomic polynomials.

Lehmer 9] asked the following question: Given $\epsilon>0$, can we find a polynomial $P(x) \in \mathbb{Z}[x]$ such that $0<m(P)<\epsilon$ ?

This question is still open 1 . The smallest known measure greater than 0 is that of a polynomial that he found in his 1933 paper:

$$
m\left(x^{10}+x^{9}-x^{7}-x^{6}-x^{5}-x^{4}-x^{3}+x+1\right)=0.1623576120 \ldots
$$

A polynomial $P(x)$ is said to be reciprocal if $P(x)= \pm x^{d} P\left(x^{-1}\right)$ where $d=$ $\operatorname{deg} P$. Notice that the above polynomial is reciprocal. Lehmer's question was answered negatively by Breusch 2 in [1] for nonreciprocal polynomials.

Lehmer's question has attracted considerable attention in the last few decades, as it has connections beyond number theory, such as entropies of dynamical systems and to polynomial knot invariants.

In this note, we explore the analogue of Lehmer's question for $m_{k}$ for $k>1$. We investigate lower bounds and limit points for higher Mahler measures and the value of $m_{2}$ and $m_{3}$ at cyclotomic polynomials.

Our main results are the following:

Theorem 4 If $P(x) \in \mathbb{Z}[x]$ is not a monomial, then for any $h \geq 1$,

$$
m_{2 h}(P) \geq \begin{cases}\left(\frac{\pi^{2}}{12}\right)^{h}, & \text { if } P(x) \text { is reciprocal, } \\ \left(\frac{\pi^{2}}{48}\right)^{h}, & \text { if } P(x) \text { is non-reciprocal. }\end{cases}
$$

This theorem is significant because the lower bound it provides is general and unconditional. Unlike well-known results regarding the lower bound for $m(P)$, the above theorem is not restricted by the behavior of the coefficients, degrees, or the reducibility properties of $P(x)$. In particular, this result implies that Lehmer's question has a negative answer for $m_{2 h}$.

A careful study of the proof of Theorem 4 reveals that $m_{2}(P)$ for $P$ reciprocal is minimized when $P(x)$ is a product of monomials and cyclotomic polynomials. Therefore, it is of interest to find out explicit values of 2 -higher Mahler measures of cyclotomic polynomials. In this direction, we prove the following theorem.

Theorem 5 For a positive integer $n$, let $\phi_{n}(x)$ denote the $n$-th cyclotomic polynomial and $\varphi$ Euler's function. Then

$$
m\left(\phi_{m}(x), \phi_{n}(x)\right)=\frac{\pi^{2}}{12} \frac{(m, n) \varphi([m, n])(-1)^{r(m)+r(n)} 2^{r((m, n))}}{[m, n]^{2}} \prod_{p \mid m n, p \nmid(m, n)} p,
$$

1 See [13] for a recent general survey on the status of this problem.

2 Later Smyth worked on this problem independently in 12 and found the best possible constant. 
where $r(x)$ denotes the number of distinct prime divisors of $x$ and the product is taken over prime numbers $p$. In particular, for $m=n$, we get

$$
m_{2}\left(\phi_{n}(x)\right)=\frac{\pi^{2}}{12} \frac{\varphi(n) 2^{r(n)}}{n} .
$$

This theorem allows us to compute $m_{2}(P)$ for $P$ any product of cyclotomic polynomials. This naturally leads us to investigate the 3-higher Mahler measure of such polynomials. We therefore prove the following theorem which relates $m_{3}(P)$ to $\zeta(3)$ and the polylogarithm.

Theorem 6 If $P(x)$ has all its roots on the unit circle, in other words, if $P(x)$ has the form

$$
P(x)=\prod_{j=1}^{n}\left(x-e^{2 \pi i \alpha_{j}}\right),
$$

with $0 \leq \alpha_{1} \leq \cdots \leq \alpha_{n}<1$, then

$$
\begin{aligned}
m_{3}(P)= & -\frac{3}{2} n^{2} \zeta(3)-3 n \sum_{1 \leq k<l \leq n} C_{3}\left(2 \pi\left(\alpha_{l}-\alpha_{k}\right)\right) \\
& -3 \pi \sum_{1 \leq k<l \leq n} S_{2}\left(2 \pi\left(\alpha_{l}-\alpha_{k}\right)\right)\left(n\left(\alpha_{l}-\alpha_{k}\right)-(l-k)\right),
\end{aligned}
$$

where

$$
C_{\ell}(t)=\sum_{n=1}^{\infty} \frac{\cos (n t)}{n^{\ell}} \quad \text { and } \quad S_{\ell}(t)=\sum_{n=1}^{\infty} \frac{\sin (n t)}{n^{\ell}}
$$

are the Clausen functions given by real and imaginary parts of the classical polylogarithm $\mathrm{Li}_{\ell}\left(e^{2 \pi i t}\right)$ defined by

$$
\operatorname{Li}_{\ell}(z)=\sum_{n=1}^{\infty} \frac{z^{n}}{n^{\ell}}
$$

in the unit disk.

Lehmer's question can be rephrased as whether 0 is a limit point for values of $m$. We generalize Lehmer's question by asking if 0 is a limit point for values of $m_{2 k+1}$ for $k \geq 1$. In this context, we prove the following.

Theorem 7 Let $P_{n}(x)=\frac{x^{n}-1}{x-1}$. For $h \geq 1$ fixed,

$$
\lim _{n \rightarrow \infty} m_{2 h+1}\left(P_{n}\right)=0
$$

Moreover, this sequence is nonconstant. 
We obtain, in this way, a positive answer for Lehmer's question for $m_{2 h+1}$.

Section 2 contains a proof of Theorem 4, which relies upon a lower bound for $m_{2}$ for products of cyclotomic polynomials. We obtain some explicit formulae for $m_{2}$ for cyclotomic polynomials and their products in Section 3 , thereby proving Theorem 5. Section 4 contains some partial results towards $m_{3}$ for cyclotomic polynomials. In particular we prove Theorem 6 in this section. Section 5 presents results about limiting points for $m_{k}$. We first consider an $m_{3}$-version for Theorem 7 in 5.1. A fundamental ingredient in the proof of Theorem 7 is a theorem of Boyd and Lawton which shows that the Mahler measure of a multivariable polynomial arises as a limit of Mahler measures of polynomials of one variable. In 5.2 we discuss a generalization of Boyd-Lawton theorem and prove the limit of Theorem 7 In Section 5.3 we prove that these sequences are non identically zero. Finally, Section 6 includes a discussion about future questions and a table with values of $m_{2}(P)$ for the reciprocal non-cyclotomic polynomials $P$ of degree less than or equal to 14 and $m(P)<0.25$. We observe that all the polynomials in the table have lower values of $m_{2}$ than Lehmer's degree 10 polynomial.

\section{A lower bound for $2 h$-Mahler measures}

In this section, we prove Theorem 4 . In order to do that, we first find a lower bound for $m_{2}$ of products of cyclotomic polynomials.

Theorem 8 If $P(x)$ is a product of cyclotomic polynomials and monomials, but is not a single monomial, then

$$
m_{2}(P) \geq \frac{\pi^{2}}{12}
$$

Before proving this, recall the following theorem from [6] (Theorem 7):

Theorem 9 For $0 \leq \alpha \leq 1$,

$$
m\left(1-x, 1-e^{2 \pi i \alpha} x\right)=\frac{\pi^{2}}{2}\left(\alpha^{2}-\alpha+\frac{1}{6}\right) .
$$

We also need the following property:

Lemma 10 If $P(x)$ has all its roots on the unit circle, in other words, if $P(x)$ has the form

$$
P(x)=\prod_{j=1}^{n}\left(x-e^{2 \pi i \alpha_{j}}\right)
$$

with $0 \leq \alpha_{j}<1$, then

$$
m_{2}(P)=\frac{\pi^{2}}{2} \sum_{1 \leq j, k \leq n}\left(\left(\alpha_{j}-\alpha_{k}\right)^{2}-\left|\alpha_{j}-\alpha_{k}\right|+\frac{1}{6}\right) .
$$


Proof. By applying Theorem 9, we can express $m_{2}(P)$ in terms of the arguments $\alpha_{i}$ :

$$
\begin{aligned}
m_{2}(P) & =\sum_{1 \leq j, k \leq n} m\left(1-e^{2 \pi i \alpha_{j}} x, 1-e^{2 \pi i \alpha_{k}} x\right)=\sum_{1 \leq j, k \leq n} m\left(1-x, 1-e^{2 \pi i\left|\alpha_{j}-\alpha_{k}\right|} x\right) \\
& =\frac{\pi^{2}}{2} \sum_{1 \leq j, k \leq n}\left(\left(\alpha_{j}-\alpha_{k}\right)^{2}-\left|\alpha_{j}-\alpha_{k}\right|+\frac{1}{6}\right) .
\end{aligned}
$$

Proof.[Theorem 8 : Since $\log |x|=0$ on the unit circle, the monomial factors do not change the value of $m_{2}(P)$. Thus, we may assume that $P(x)$ can be written as

$$
P(x)=(x-1)^{a}(x+1)^{b} \prod_{j=1}^{2 n}\left(x-e^{2 \pi i \alpha_{j}}\right)
$$

with $0 \leq \alpha_{1} \leq \cdots \leq \alpha_{2 n} \leq 1$ with $\alpha_{j}=1-\alpha_{2 n+1-j}$. In addition, $a, b \in\{0,1\}$ as they account for the fact that we may have an odd number of factors $x-1$ and/or $x+1$ in the product. Using that $m(x+1, x-1)=-\frac{\pi^{2}}{24}$ and Lemma 10, we obtain

$$
\begin{aligned}
m_{2}(P)= & a m_{2}(x-1)+b m_{2}(x+1)+2 a b m(x+1, x-1) \\
& +2 a m\left(x-1, \prod_{j=1}^{2 n}\left(x-e^{2 \pi i \alpha_{j}}\right)\right)+2 b m\left(x+1, \prod_{j=1}^{2 n}\left(x-e^{2 \pi i \alpha_{j}}\right)\right) \\
& +m_{2}\left(\prod_{j=1}^{2 n}\left(x-e^{2 \pi i \alpha_{j}}\right)\right) \\
= & \frac{\pi^{2}}{2}\left(\frac{a+b-a b}{6}+2 a \sum_{j=1}^{2 n}\left(\alpha_{j}^{2}-\alpha_{j}+\frac{1}{6}\right)\right. \\
& +2 b \sum_{j=1}^{2 n}\left(\left(\alpha_{j}-\frac{1}{2}\right)^{2}-\left|\alpha_{j}-\frac{1}{2}\right|+\frac{1}{6}\right)+4 n \sum_{j=1}^{2 n} \alpha_{j}^{2} \\
& \left.-\sum_{1 \leq j, k \leq 2 n}\left(2 \alpha_{j} \alpha_{k}+\left|\alpha_{j}-\alpha_{k}\right|\right)+\frac{2 n^{2}}{3}\right) \\
= & \frac{\pi^{2}}{2}\left(\frac{a+b-a b}{6}+2(a+b) \sum_{j=1}^{2 n} \alpha_{j}^{2}-2 a \sum_{j=1}^{2 n} \alpha_{j}-4 b \sum_{j=n+1}^{2 n} \alpha_{j}+b n\right. \\
& +4 n \sum_{j=1}^{2 n} \alpha_{j}^{2}-2 \sum_{1 \leq j, k \leq 2 n} \alpha_{j} \alpha_{k}-2 \sum_{j=1}^{2 n} j \alpha_{j}+2 \sum_{j=1}^{2 n}(2 n+1-j) \alpha_{j} \\
& \left.+\frac{2 n(n+a+b)}{3}\right) .
\end{aligned}
$$


Because of $\alpha_{j}=1-\alpha_{2 n+1-j}$, we have that $\sum_{j=1}^{2 n} \alpha_{j}=n$. This implies that

$$
\begin{aligned}
m_{2}(P)= & \frac{\pi^{2}}{2}\left(2(a+b+2 n) \sum_{j=1}^{2 n} \alpha_{j}^{2}-4 b \sum_{j=n+1}^{2 n} \alpha_{j}-4 \sum_{j=1}^{2 n} j \alpha_{j}\right. \\
& \left.+2 n(n+1)+\frac{n(2 n-4 a+5 b)}{3}+\frac{a+b-a b}{6}\right) .
\end{aligned}
$$

Let $\alpha:=\alpha_{j}$ with $1 \leq j \leq n$ so that $0 \leq \alpha \leq \frac{1}{2}$. In this case, we define

$$
\begin{aligned}
g(\alpha) & :=2(a+b+2 n)\left(\alpha^{2}+(1-\alpha)^{2}\right)-4(j \alpha+(2 n+1-j+b)(1-\alpha)) \\
& =4(a+b+2 n) \alpha^{2}+4(1-2 j-a) \alpha+4 j+2 a-2 b-4 n-4 .
\end{aligned}
$$

Since we have a quadratic equation, the minimum of $g(\alpha)$ is achieved with $\alpha=\frac{a+2 j-1}{2(a+b+2 n)}$. Thus

$$
g(\alpha) \geq-\frac{(a+2 j-1)^{2}}{a+b+2 n}+4 j+2 a-2 b-4 n-4
$$

We use the bound on $g(\alpha)$ in order to obtain

$$
\begin{aligned}
\frac{2}{\pi^{2}} m_{2}(P) \geq & \sum_{j=1}^{n}\left(-\frac{(a+2 j-1)^{2}}{a+b+2 n}+4 j+2 a-2 b-4 n-4\right) \\
& +2 n(n+1)+\frac{n(2 n-4 a+5 b)}{3}+\frac{a+b-a b}{6} \\
= & \sum_{j=1}^{n}\left(-\frac{4 j^{2}}{a+b+2 n}+\frac{4(b+2 n+1) j}{a+b+2 n}-\frac{(a-1)^{2}}{a+b+2 n}\right) \\
& -\frac{n(4 n-2 a+b+6)}{3}+\frac{a+b-a b}{6} \\
= & -\frac{2 n(n+1)(2 n+1)}{3(a+b+2 n)}+\frac{2(b+2 n+1) n(n+1)}{a+b+2 n}-\frac{(a-1)^{2} n}{a+b+2 n} \\
& -\frac{n(4 n-2 a+b+6)}{3}+\frac{a+b-a b}{6} \\
= & \frac{2 n(n+1)(3 b+4 n+2)}{3(a+b+2 n)}-\frac{(a-1)^{2} n}{a+b+2 n} \\
& -\frac{n(4 n-2 a+b+6)}{3}+\frac{a+b-a b}{6} \\
= & \frac{1}{6},
\end{aligned}
$$

where the last equality is valid for any of the four cases with $a, b \in\{0,1\}$.

Thus,

$$
m_{2}(P) \geq \frac{\pi^{2}}{12} \cong 0.8224670334 \ldots
$$


Remark 11 Observe that the previous proof only uses the fact that $P$ is reciprocal with roots on the unit circle. Therefore, Theorem 8 applies to this family of polynomials.

In order to prove Theorem 4, we extend Theorem 8 to reciprocal polynomials:

Theorem 12 If $P(x) \in \mathbb{Z}[x]$ is reciprocal, then

$$
m_{2}(P) \geq \frac{\pi^{2}}{12}
$$

We will need the following result which is Remark 9 in [6]:

Lemma 13 For $a, b \in \mathbb{C}$,

$$
m(1-a x, 1-b x)= \begin{cases}\frac{1}{2} \operatorname{Re}_{2}(a \bar{b}) & \text { if }|a|,|b| \leq 1, \\ \frac{1}{2} \operatorname{Re}^{2}(b / \bar{a}) & \text { if }|a| \geq 1,|b| \leq 1, \\ \frac{1}{2} \operatorname{Re} \operatorname{Li}_{2}(1 / \bar{a} b)+\log |a| \log |b| & \text { if }|a|,|b| \geq 1,\end{cases}
$$

where $\mathrm{Li}_{2}$ is the dilogarithm function.

Proposition 14 Let $\tau_{1}, \ldots, \tau_{M}$ be fixed real numbers in $[0,1)$ and $c_{1}, \ldots, c_{M}>$ 0 . The function

$$
f\left(y_{1}, \ldots, y_{M}\right)=\sum_{j=1}^{M} c_{i} \sum_{n=1}^{\infty} \frac{y_{j}^{n} \cos \left(2 \pi n \tau_{i}\right)}{n^{2}}
$$

attains its minimum in $[0,1]^{M}$ at a point where $y_{i} \in\{0,1\}$ for each $i$.

Proof. For a fixed $\tau \in[0,1)$, we first study the function

$$
g(y)=\sum_{n=1}^{\infty} \frac{y^{n} \cos (2 \pi n \tau)}{n^{2}}
$$

in the interval $[0,1]$. In this interval, $g(y)$ attains its minimum either at the end points or when $g^{\prime}(y)=0$. However,

$$
g^{\prime}(y)=\frac{1}{y} \sum_{n=1}^{\infty} \frac{y^{n} \cos (2 \pi n \tau)}{n}=-\frac{1}{y} \log \left|1-y e^{2 \pi i \tau}\right| .
$$

Thus, we get a critical point when $\left|1-y e^{2 \pi i \tau}\right|=1$, that is, when

$$
(1-y \cos (2 \pi \tau))^{2}+(y \sin (2 \pi \tau))^{2}=1
$$


and therefore, $y_{0}=2 \cos (2 \pi \tau)$. We need to determine what kind of point $y_{0}$ is. Observe that

$$
g^{\prime \prime}(y)=\frac{1}{y^{2}} \log \left|1-y e^{2 \pi i \tau}\right|+\frac{1}{y^{2}} \operatorname{Re}\left(\frac{y e^{2 \pi i \tau}}{1-y e^{2 \pi i \tau}}\right) .
$$

Thus,

$$
g^{\prime \prime}\left(y_{0}\right)=\frac{1}{y_{0}^{2}} \operatorname{Re}\left(y_{0} e^{2 \pi i \tau}\left(1-y_{0} e^{-2 \pi i \tau}\right)\right)=\frac{1}{y_{0}^{2}}\left(y_{0} \cos (2 \pi \tau)-y_{0}^{2}\right)=-\frac{1}{2}<0 .
$$

Then, $y_{0}$ is a (local) maximum point for $g(y)$. Therefore, the minimum for $g(y)$ in $[0,1]$ is either at $y=0$ or $y=1$. Since each $c_{i}>0$, we conclude that in the interval $[0,1]^{M}, f\left(y_{1}, \ldots, y_{M}\right)$ attains its minimum at a point where each $y_{i}$ is either 0 or 1 .

Remark 15 From the above analysis it also follows that if $f\left(y_{1}, \ldots, y_{M}\right) \geq$ $f\left(a_{1}, \ldots, a_{M}\right)$ for all $\left(y_{1}, \ldots, y_{M}\right) \in(0,1)^{M}$, then each $a_{i} \in\{0,1\}$.

Proof.[Theorem 12. Let $P(x)$ be a reciprocal polynomial in $\mathbb{Z}[x]$. If $P$ is not monic, we can write $P(x)=C Q(x)$ with $C \in \mathbb{Z}$ and

$$
m_{2}(P)=\log ^{2} C+2 \log C m(Q)+m_{2}(Q) \geq m_{2}(Q),
$$

where we are using that $C \geq 1$ in the inequality.

Therefore we can assume that $P$ is monic. Thus we write

$$
P(x)=(x-1)^{a}(x+1)^{b} \prod_{j=1}^{J}\left(x-r_{j}\right)\left(x-r_{j}^{-1}\right)
$$

where $\left|r_{j}\right| \leq 1$. We write $r_{j}=\rho_{j} e^{2 \pi i \mu_{j}}$ with $0 \leq \rho_{j} \leq 1$. Here we need to clarify what for $\rho_{j}=0$ the product $\left(x-r_{j}\right)\left(x-r_{j}^{-1}\right)$ should be interpreted as the product $x \cdot 1$. It is important to understand that Lemma 13 is still valid in these cases since $\mathrm{Li}_{2}(0)=0$.

In addition, $a, b \in\{0,1\}$ as they account for the fact that we may have an odd number of factors $x-1$ and/or $x+1$ in the product.

Then

$$
\begin{aligned}
m_{2}(P)= & a m_{2}(x-1)+b m_{2}(x+1)+2 a b m(x+1, x-1)+2 a \sum_{j=1}^{J} m\left(x-1, x-r_{j}\right) \\
& +2 b \sum_{j=1}^{J} m\left(x-1, x-r_{j}^{-1}\right)+\sum_{1 \leq j_{1}, j_{2} \leq J} m\left(x-r_{j_{1}}, x-r_{j_{2}}\right) \\
& +\sum_{1 \leq j_{1}, j_{2} \leq J} m\left(x-r_{j_{1}}^{-1}, x-r_{j_{2}}^{-1}\right)+2 \sum_{1 \leq j_{1}, j_{2} \leq J} m\left(x-r_{j_{1}}, x-r_{j_{2}}^{-1}\right) .
\end{aligned}
$$


Using that $m(x+1, x-1)=-\frac{\pi^{2}}{24}$ and applying Lemma 13 ,

$$
\begin{aligned}
m_{2}(P)= & (a+b-a b) \frac{\pi^{2}}{12}+(a+b) \sum_{j=1}^{J} \operatorname{Re}^{2} \operatorname{Li}_{2}\left(r_{j}\right) \\
& +\sum_{1 \leq j_{1}, j_{2} \leq J}\left(2 \operatorname{Re} \operatorname{Li}_{2}\left(r_{j_{1}} \overline{r_{j_{2}}}\right)+\log \left|r_{j_{1}}\right| \log \left|r_{j_{2}}\right|\right) .
\end{aligned}
$$

By writing the dilogarithm in terms of its power series, we get

$$
\begin{aligned}
m_{2}(P)= & (a+b-a b) \frac{\pi^{2}}{12}+m(P)^{2}+(a+b) \sum_{j=1}^{J} \sum_{n=1}^{\infty} \frac{\rho_{j}^{n} \cos \left(2 \pi n \mu_{j}\right)}{n^{2}} \\
& +2 \sum_{1 \leq j_{1}, j_{2} \leq J} \sum_{n=1}^{\infty} \frac{\rho_{j_{1}}^{n} \rho_{j_{2}}^{n} \cos \left(2 \pi n\left(\mu_{j_{1}}-\mu_{j_{2}}\right)\right)}{n^{2}} .
\end{aligned}
$$

Thus, the problem of minimizing $m_{2}(P)$ reduces to the problem of minimizing the terms in the above expression. First let us fix the arguments $\mu_{j}$. As a consequence of Proposition [14 and Remark 15. we see that the last term involving a series reaches its minimum when $\rho_{j} \in\{0,1\}$. This condition also minimizes the other term involving a series, although that term can be ignored if $a=b=0$. This means that there are no roots with absolute value greater than 1. This also minimizes the first term $m(P)^{2}$ which is nonnegative for $P(x)$ monic and zero if all the roots of $P(x)$ are of absolute value (less than or) equal to 1 . Now if we allow the arguments $\mu_{j}$ to vary, the minimum $m_{2}(P)$ is still attained when all the roots have absolute value in $\{0,1\}$. Since $P$ is reciprocal and its roots have absolute value 1 , we can apply Theorem 8 and Remark 11 to conclude that

$$
m_{2}(P) \geq \frac{\pi^{2}}{12}
$$

In order to prove Theorem 4 we need to say what happens when $P$ is not reciprocal.

Lemma 16 If $P(x) \in \mathbb{Z}[x]$ is nonreciprocal, then

$$
m_{2}(P) \geq \frac{\pi^{2}}{48}
$$

Proof. Let $d=\operatorname{deg} P$, and consider $Q(x)=x^{d} P\left(x^{-1}\right)$. Thus $P(x) Q(x) \in \mathbb{Z}[x]$ is reciprocal. Moreover, $m_{2}(P)=m_{2}(Q)=m(P, Q)$, thus,

$$
m_{2}(P Q)=m_{2}(P)+2 m(P, Q)+m_{2}(Q)=4 m_{2}(P) .
$$

We obtain the desired bound by applying Theorem 12 to $P Q$. 
Remark 17 While the inequality in Theorem 12 is sharp $\left(\right.$ as $\left.m_{2}(x-1)=\frac{\pi^{2}}{12}\right)$, we do not know what happens with the inequality in Lemma 16. The best polynomial we were able to find is

$$
m_{2}\left(x^{3}+x+1\right) \cong 0.3275495729 \ldots,
$$

while $\frac{\pi^{2}}{48} \cong 0.2056167583 \ldots$

We will use the bound for $m_{2}(P)$ in order to find a bound for $m_{2 h}(P)$.

Proposition 18 For any nonzero polynomial $P(x) \in \mathbb{C}[x]$,

1.

$$
m_{2 h}(P) \geq m_{2}(P)^{h},
$$

2.

$$
m_{2 h}(P) \geq m(P)^{2 h} .
$$

Proof. Part 1. For any positive integer $h$, let $f$ and $g$ be functions such that

$$
\frac{1}{2 \pi i} \int_{|x|=1}|f|^{h} \frac{d x}{x}<\infty \quad \text { and } \quad \frac{1}{2 \pi i} \int_{|x|=1}|g|^{h /(h-1)} \frac{d x}{x}<\infty .
$$

Then, by Hölder's inequality, we get that

$$
\left(\frac{1}{2 \pi i} \int_{|x|=1}|f g| \frac{d x}{x}\right)^{h} \leq\left(\frac{1}{2 \pi i} \int_{|x|=1}|f|^{h} \frac{d x}{x}\right)\left(\frac{1}{2 \pi i} \int_{|x|=1}|g|^{h /(h-1)} \frac{d x}{x}\right)^{h-1} .
$$

In particular, taking $f(x)=\log ^{2}|P(x)|$ and $g(x)=1$, we get that

$$
m_{2}(P)^{h} \leq m_{2 h}(P) \text {. }
$$

Part 2. On the other hand, by taking $f(x)=\log |P(x)|$ and $g(x)=1$, and taking $2 h$ instead of $h$ in (2) we get that

$$
m(P)^{2 h} \leq m_{2 h}(P) .
$$

Proof. [Theorem 4. By combining Theorem 12, Lemma 16, and Part 1 of Proposition 18 we obtain that,

$$
m_{2 h}(P) \geq m_{2}(P)^{h} \geq \begin{cases}\left(\frac{\pi^{2}}{12}\right)^{h}, & \text { if } P(x) \text { is reciprocal }, \\ \left(\frac{\pi^{2}}{48}\right)^{h}, & \text { if } P(x) \text { is non-reciprocal. }\end{cases}
$$


Remark 19 By Part 2 of Proposition 18, if we assume that the lowest positive value of $m(P)$ is for Lehmer's degree 10 polynomial, then for any $P(x) \in \mathbb{Z}[x]$ with $m(P)>0$,

$$
m_{2}(P) \geq(0.1623576120 \ldots)^{2} \cong 0.0263599941 \ldots
$$

However, Theorem 4 provides us with an unconditional and stronger lower bound $0.2056167583 \ldots$ for $m_{2}(P)$ (and $m_{2 h}(P)$ ).

Analogously, we can use the result of Smyth to find a different bound in Lemma [16. Smyth [12] proved that for $P \in \mathbb{Z}[x]$ nonreciprocal,

$$
m(P) \geq m\left(x^{3}-x-1\right) \cong 0.2811995743 \ldots
$$

This can be combined with Part 2 of Proposition 18 to obtain

$$
m_{2}(P) \geq(0.2811995743 \ldots)^{2} \cong 0.0790732005 \ldots,
$$

but this bound is less than $\frac{\pi^{2}}{48}$ and therefore weaker.

\section{Explicit formulae for 2-Mahler measures of cyclotomic polynomials}

While the classical Mahler measure for products of cyclotomic polynomials is uninteresting, we have seen that the same is not true for higher Mahler measures. In this section we show how to evaluate $m_{2}(P)$ for such polynomials. We notice that any product of cyclotomic polynomials can be written as

$$
P(x)=\prod_{i=1}^{N}\left(x^{d_{i}}-1\right)^{e_{i}}
$$

where we allow negative exponents. Therefore, we can compute $m_{2}(P)$ if we understand $m\left(x^{a}-1, x^{b}-1\right)$.

We start by proving the following useful result, which is also of independent interest.

Proposition 20 For any two positive coprime integers a and b,

$$
S(a, b):=\sum_{j=0}^{a-1} \sum_{k=0}^{b-1}\left|\frac{k}{b}-\frac{j}{a}\right|=\frac{2 a^{2} b^{2}-3 a b+a^{2}+b^{2}-1}{6 a b} .
$$

Proof. First we observe that the term inside the absolute value is positive when $\frac{j}{a}<\frac{k}{b}$. For fixed $j$, this happens for $k=b-1, \ldots,\left\lfloor\frac{b j}{a}\right\rfloor+1$, that is, for $b-\left\lfloor\frac{b j}{a}\right\rfloor-1$ values of $k$. On the other hand, it is negative when $k=$ $0, \ldots,\left\lfloor\frac{b j}{a}\right\rfloor$, that is, for $\left\lfloor\frac{b j}{a}\right\rfloor+1$ values of $k$. Thus, $\frac{j}{a}$ appears with negative sign for $b-\left\lfloor\frac{b j}{a}\right\rfloor-1$ values of $k$ and with positive sign for $\left\lfloor\frac{b j}{a}\right\rfloor+1$ values of 
$k$. Putting this into the equation (together with the same analysis for $k$ ), we obtain,

$$
\begin{aligned}
S(a, b) & =\sum_{j=0}^{a-1} \frac{j}{a}\left(2\left\lfloor\frac{b j}{a}\right\rfloor+2-b\right)+\sum_{k=0}^{b-1} \frac{k}{b}\left(2\left\lfloor\frac{a k}{b}\right\rfloor+2-a\right) \\
& =\frac{(2-b)(a-1)}{2}+\frac{(2-a)(b-1)}{2}+\frac{2}{a} \sum_{j=0}^{a-1} j\left\lfloor\frac{b j}{a}\right\rfloor+\frac{2}{b} \sum_{k=0}^{b-1} k\left\lfloor\frac{a k}{b}\right\rfloor .
\end{aligned}
$$

Assume without loss of generality that $a>b$. Let $j_{l}$ be such that $\left\lfloor\frac{b j_{l}}{a}\right\rfloor=l$ and $\left\lfloor\frac{b\left(j_{l}-1\right)}{a}\right\rfloor=l-1$. Then $0=j_{0}<j_{1}<\cdots<j_{b-1}<j_{b}=a$. Thus,

$$
\begin{aligned}
\frac{2}{a} \sum_{j=0}^{a-1} j\left\lfloor\frac{b j}{a}\right\rfloor+\frac{2}{b} \sum_{k=0}^{b-1} k\left\lfloor\frac{a k}{b}\right\rfloor & =\frac{2}{a} \sum_{l=1}^{b} \sum_{j=j_{l-1}}^{j_{l}-1} j\left\lfloor\frac{b j}{a}\right\rfloor+\frac{2}{b} \sum_{k=0}^{b-1} k\left\lfloor\frac{a k}{b}\right\rfloor \\
& =\frac{2}{a} \sum_{l=1}^{b}(l-1) \sum_{j=j_{l-1}}^{j_{l}-1} j+\frac{2}{b} \sum_{k=0}^{b-1} k\left\lfloor\frac{a k}{b}\right\rfloor \\
& =\frac{2}{a} \sum_{l=1}^{b}(l-1)\left(\left(\begin{array}{c}
j_{l} \\
2
\end{array}\right)-\left(\begin{array}{c}
j_{l-1} \\
2
\end{array}\right)\right)+\frac{2}{b} \sum_{k=0}^{b-1} k\left\lfloor\frac{a k}{b}\right\rfloor \\
& =\frac{2}{a}\left((b-1)\left(\begin{array}{c}
a \\
2
\end{array}\right)-\sum_{l=1}^{b-1}\left(\begin{array}{c}
j_{l} \\
2
\end{array}\right)\right)+\frac{2}{b} \sum_{k=0}^{b-1} k\left\lfloor\frac{a k}{b}\right\rfloor
\end{aligned}
$$

Notice that for $l>0$,

$$
\frac{b\left(j_{l}-1\right)}{a}<l<\frac{b j_{l}}{a}
$$

which implies that $j_{l}=\left\lfloor\frac{a l}{b}\right\rfloor+1$. Thus, the above computation equals

$$
(a-1)(b-1)-\frac{1}{a} \sum_{l=0}^{b-1}\left(\left\lfloor\frac{a l}{b}\right\rfloor^{2}+\left\lfloor\frac{a l}{b}\right\rfloor\right)+\frac{2}{b} \sum_{k=0}^{b-1} k\left\lfloor\frac{a k}{b}\right\rfloor .
$$

Because of $\sum_{l=0}^{b-1}\left\lfloor\frac{a l}{b}\right\rfloor=\frac{(a-1)(b-1)}{2}$, the above equals

$$
\begin{aligned}
& (a-1)(b-1)-\frac{(a-1)(b-1)}{2 a}-\frac{1}{a} \sum_{k=0}^{b-1}\left\lfloor\frac{a k}{b}\right\rfloor^{2}+\frac{2}{b} \sum_{k=0}^{b-1} k\left\lfloor\frac{a k}{b}\right\rfloor \\
= & \frac{(a-1)(2 a-1)(b-1)}{2 a}-\frac{1}{a} \sum_{k=0}^{b-1}\left(\left\lfloor\frac{a k}{b}\right\rfloor-\frac{a k}{b}\right)^{2}+\frac{1}{a} \sum_{k=0}^{b-1} \frac{a^{2} k^{2}}{b^{2}} .
\end{aligned}
$$

Observe that because $(a, b)=1$, as the term $k$ runs through all the residues modulo $b$, so does the term $b\left(\frac{a k}{b}-\left\lfloor\frac{a k}{b}\right\rfloor\right)$. Thus, the above expression is equal 
to

$$
\begin{aligned}
& \frac{(a-1)(2 a-1)(b-1)}{2 a}-\frac{1}{a b^{2}} \sum_{k=0}^{b-1} k^{2}+\frac{a}{b^{2}} \sum_{k=0}^{b-1} k^{2} \\
= & \frac{(a-1)(2 a-1)(b-1)}{2 a}+\frac{\left(a^{2}-1\right)(b-1)(2 b-1)}{6 a b} .
\end{aligned}
$$

Finally,

$S(a, b)=\frac{(a-1)+(b-1)-2(a-1)(b-1)}{2}+\frac{(a-1)(b-1)(8 a b-a-b-1)}{6 a b}$

From the above proposition, we deduce the following theorem:

Theorem 21 For any two positive integers $a$ and $b$,

$$
m\left(x^{a}-1, x^{b}-1\right)=\frac{\pi^{2}}{12} \frac{(a, b)^{2}}{a b} .
$$

Proof. First assume that $(a, b)=1$. Applying Lemma 10, we observe that

$$
\begin{aligned}
m\left(x^{a}-1, x^{b}-1\right) & =\frac{\pi^{2}}{2} \sum_{j=0}^{a-1} \sum_{k=0}^{b-1}\left(\left|\frac{k}{b}-\frac{j}{a}\right|^{2}-\left|\frac{k}{b}-\frac{j}{a}\right|+\frac{1}{6}\right) \\
& =\frac{\pi^{2}}{2} \sum_{j=0}^{a-1} \sum_{k=0}^{b-1}\left(\frac{k^{2}}{b^{2}}+\frac{j^{2}}{a^{2}}-\frac{2 j k}{a b}+\frac{1}{6}-\left|\frac{k}{b}-\frac{j}{a}\right|\right) .
\end{aligned}
$$

Therefore, applying Proposition 20, we have

$$
\begin{aligned}
m\left(x^{a}-1, x^{b}-1\right) & =\frac{\pi^{2}}{2}\left(\frac{2 a^{2} b^{2}+a^{2}+b^{2}-3 a b}{6 a b}-\frac{2 a^{2} b^{2}-3 a b+a^{2}+b^{2}-1}{6 a b}\right) \\
& =\frac{\pi^{2}}{12 a b} .
\end{aligned}
$$

For general $a$ and $b$ it suffices to notice that the change of variables $y=x^{(a, b)}$ will not affect the Mahler measure, and thus

$$
m\left(x^{a}-1, x^{b}-1\right)=m\left(x^{\frac{a}{(a, b)}}-1, x^{\frac{b}{(a, b)}}-1\right) .
$$

From Theorem 21 we deduce the following proposition. 
Proposition 22 For a positive integer $n$, let $\phi_{n}(x)$ denote the $n$-th cyclotomic polynomial and $\mu$ be the Möbius function. For any two positive integers $m$ and $n$,

$$
m\left(\phi_{m}(x), \phi_{n}(x)\right)=\frac{\pi^{2}}{12} \sum_{d_{1}\left|m, d_{2}\right| n} \mu\left(\frac{m}{d_{1}}\right) \mu\left(\frac{n}{d_{2}}\right) \frac{\left(d_{1}, d_{2}\right)^{2}}{d_{1} d_{2}} .
$$

Proof. We recall that for any positive integer $n$,

$$
x^{n}-1=\prod_{d \mid n} \phi_{d}(x) .
$$

Thus, by the multiplicative Möbius inversion formula, we get that

$$
\phi_{n}(x)=\prod_{d \mid n}\left(x^{d}-1\right)^{\mu(n / d)} .
$$

From the above and from Theorem 21,

$$
\begin{aligned}
m\left(\phi_{m}(x), \phi_{n}(x)\right) & =\sum_{d_{1}\left|m, d_{2}\right| n} \mu\left(\frac{m}{d_{1}}\right) \mu\left(\frac{n}{d_{2}}\right) m\left(x^{d_{1}}-1, x^{d_{2}}-1\right) \\
& =\frac{\pi^{2}}{12} \sum_{d_{1}\left|m, d_{2}\right| n} \mu\left(\frac{m}{d_{1}}\right) \mu\left(\frac{n}{d_{2}}\right) \frac{\left(d_{1}, d_{2}\right)^{2}}{d_{1} d_{2}} .
\end{aligned}
$$

Proposition 23 Let $p$ be a positive prime. We have the following transformations

1. For $k \geq l \geq 1$ and $p \nmid m n$,

$$
m\left(\phi_{p^{k} m}(x), \phi_{p^{l} n}(x)\right)=\frac{2}{p^{k-l}}\left(1-\frac{1}{p}\right) m\left(\phi_{m}(x), \phi_{n}(x)\right) .
$$

2. For $k \geq 1$ and $p \nmid m n$,

$$
m\left(\phi_{p^{k} m}(x), \phi_{n}(x)\right)=-\frac{1}{p^{k-1}}\left(1-\frac{1}{p}\right) m\left(\phi_{m}(x), \phi_{n}(x)\right) .
$$

Proof. Part 1. Using Proposition 22, we have

$$
m\left(\phi_{p^{k} m}(x), \phi_{p^{l} n}(x)\right)=\frac{\pi^{2}}{12} \sum_{d_{1}\left|p^{k} m, d_{2}\right| p^{l} n} \mu\left(\frac{p^{k} m}{d_{1}}\right) \mu\left(\frac{p^{l} n}{d_{2}}\right) \frac{\left(d_{1}, d_{2}\right)^{2}}{d_{1} d_{2}} .
$$

It is clear that only the terms with $p^{k-1} \mid d_{1}$ and $p^{l-1} \mid d_{2}$ are nonzero, since otherwise the Möbius function factors yield zero. We write $d_{1}=p^{k-1} e_{1}$ and $d_{2}=p^{l-1} e_{2}$. Thus 


$$
m\left(\phi_{p^{k} m}(x), \phi_{p^{l} n}(x)\right)=\frac{\pi^{2}}{12} \sum_{e_{1}\left|p m, e_{2}\right| p n} \mu\left(\frac{p m}{e_{1}}\right) \mu\left(\frac{p n}{e_{2}}\right) \frac{\left(e_{1}, e_{2}\right)^{2}}{e_{1} e_{2} p^{k-l}} .
$$

If $p$ divides both $e_{1}$ and $e_{2}$ or $p$ does not divide either of them, we get terms of the form

$$
\mu\left(\frac{m}{f_{1}}\right) \mu\left(\frac{n}{f_{2}}\right) \frac{\left(f_{1}, f_{2}\right)^{2}}{f_{1} f_{2} p^{k-l}}
$$

with $p \nmid f_{i}$.

If, on the other hand, $p$ divides exactly one of the $e_{i}$ 's, we get terms of the form

$$
-\mu\left(\frac{m}{f_{1}}\right) \mu\left(\frac{n}{f_{2}}\right) \frac{\left(f_{1}, f_{2}\right)^{2}}{f_{1} f_{2} p^{k-l+1}}
$$

with $p \nmid f_{i}$.

Putting all of these ideas together, we obtain

$$
\begin{aligned}
m\left(\phi_{p^{k} m}(x), \phi_{p^{l} n}(x)\right) & =\frac{\pi^{2}}{12} \frac{2}{p^{k-l}}\left(1-\frac{1}{p}\right) \sum_{f_{1}\left|m, f_{2}\right| n} \mu\left(\frac{m}{f_{1}}\right) \mu\left(\frac{n}{f_{2}}\right) \frac{\left(f_{1}, f_{2}\right)^{2}}{f_{1} f_{2}} \\
& =\frac{2}{p^{k-l}}\left(1-\frac{1}{p}\right) m\left(\phi_{m}(x), \phi_{n}(x)\right),
\end{aligned}
$$

which proves the first part of the proposition.

Part 2. Once again, by Proposition 22, we can write

$$
m\left(\phi_{p^{k} m}(x), \phi_{n}(x)\right)=\frac{\pi^{2}}{12} \sum_{d_{1}\left|p^{k} m, d_{2}\right| n} \mu\left(\frac{p^{k} m}{d_{1}}\right) \mu\left(\frac{n}{d_{2}}\right) \frac{\left(d_{1}, d_{2}\right)^{2}}{d_{1} d_{2}} .
$$

As before, it is clear that $p^{k-1} \mid d_{1}$ in the nonzero terms, and we can write $d_{1}=p^{k-1} e_{1}$.

If $p \mid e_{1}$, we obtain

$$
\mu\left(\frac{m}{f_{1}}\right) \mu\left(\frac{n}{d_{2}}\right) \frac{\left(f_{1}, d_{2}\right)^{2}}{f_{1} d_{2} p^{k}}
$$

with $p \nmid f_{1}$.

If $p \nmid e_{1}$, we obtain

$$
-\mu\left(\frac{m}{e_{1}}\right) \mu\left(\frac{n}{d_{2}}\right) \frac{\left(e_{1}, d_{2}\right)^{2}}{e_{1} d_{2} p^{k-1}}
$$

Thus,

$$
\begin{aligned}
m\left(\phi_{p^{k} m}(x), \phi_{n}(x)\right) & =-\frac{\pi^{2}}{12} \frac{1}{p^{k-1}}\left(1-\frac{1}{p}\right) \sum_{e_{1}\left|m, d_{2}\right| n} \mu\left(\frac{m}{e_{1}}\right) \mu\left(\frac{n}{d_{2}}\right) \frac{\left(e_{1}, d_{2}\right)^{2}}{e_{1} d_{2}} \\
& =-\frac{1}{p^{k-1}}\left(1-\frac{1}{p}\right) m\left(\phi_{m}(x), \phi_{n}(x)\right)
\end{aligned}
$$


proving the second part of the proposition.

Proof. [Theorem 5] We write the prime factorizations of $m$ and $n$ as $m=$ $p_{1}^{k_{1}} \ldots p_{r}^{k_{r}} q_{1}^{h_{1}} \ldots q_{u}^{h_{u}}$ and $n=p_{1}^{l_{1}} \ldots p_{r}^{l_{r}} t_{1}^{j_{1}} \ldots t_{v}^{j_{v}}$, where all the exponents are positive integers and the primes $q$ 's are different from the primes $t$ 's. Thus $r=r((m, n))$. By applying Proposition 23, we obtain

$$
\begin{aligned}
& m\left(\phi_{m}(x), \phi_{n}(x)\right) \\
= & 2^{r} \prod_{i=1}^{r}\left(\frac{1}{p_{i}^{\left|k_{i}-l_{i}\right|}}\left(1-\frac{1}{p_{i}}\right)\right)(-1)^{u+v} \prod_{i=1}^{u}\left(\frac{1}{q_{i}^{h_{i}-1}}\left(1-\frac{1}{q_{i}}\right)\right) \prod_{i=1}^{v}\left(\frac{1}{t_{i}^{j_{i}-1}}\left(1-\frac{1}{t_{i}}\right)\right) m_{2}(x-1) \\
= & \frac{\pi^{2}}{12} 2^{r}(-1)^{u+v} \prod_{i=1}^{r} \frac{p_{i}^{\min \left\{k_{i}, l_{i}\right\}}}{p_{i}^{\max \left\{k_{i}, l_{i}\right\}}} \prod_{i=1}^{u} \frac{q_{i}}{q_{i}^{h_{i}}} \prod_{i=1}^{v} \frac{t_{i}}{t_{i}^{j_{i}}} \prod_{p \mid m n}\left(1-\frac{1}{p}\right) \\
= & \frac{\pi^{2}}{12} 2^{r((m, n))}(-1)^{r(m)+r(n)} \frac{(m, n)}{[m, n]} \prod_{i=1}^{u} q_{i} \prod_{i=1}^{v} t_{i} \prod_{p \mid m n}\left(1-\frac{1}{p}\right) \\
= & \frac{\pi^{2}}{12} \frac{2^{r((m, n))}(-1)^{r(m)+r(n)}(m, n)}{[m, n]}\left(\prod_{p \mid m n, p \nmid(m, n)} p\right) \frac{\varphi([m, n])}{[m, n]} .
\end{aligned}
$$

\section{Explicit formulae for 3-Mahler measures of some particular polynomials}

In this section, we address the case of $m_{3}(P)$ for $P$ a product of cyclotomic polynomials. Our starting point is Remark 10 from [6], which is the following statement:

Proposition 24 We have

$$
\begin{aligned}
m\left(1-x, 1-e^{2 \pi i \alpha} x, 1-e^{2 \pi i \beta} x\right)= & -\frac{1}{4} \sum_{1 \leq k, l} \frac{\cos 2 \pi((k+l) \beta-l \alpha)}{k l(k+l)} \\
& -\frac{1}{4} \sum_{1 \leq k, m} \frac{\cos 2 \pi((k+m) \alpha-m \beta)}{k m(k+m)} \\
& -\frac{1}{4} \sum_{1 \leq l, m} \frac{\cos 2 \pi(l \alpha+m \beta)}{l m(l+m)} .
\end{aligned}
$$

First, we express the above formula in terms of Clausen functions. 
Proposition 25 For $0 \leq \alpha, \beta<1$, we have

$$
\begin{aligned}
2 m\left(1-x, 1-e^{2 \pi i \alpha} x, 1-e^{2 \pi i \beta} x\right)= & S_{1}(2 \pi(\beta-\alpha)) S_{2}(2 \pi \beta)+S_{2}(2 \pi(\beta-\alpha)) S_{1}(2 \pi \beta) \\
& -C_{3}(2 \pi \alpha)+S_{1}(2 \pi(\alpha-\beta)) S_{2}(2 \pi \alpha) \\
& +S_{2}(2 \pi(\alpha-\beta)) S_{1}(2 \pi \alpha)-C_{3}(2 \pi \beta) \\
& +S_{1}(2 \pi \alpha) S_{2}(2 \pi \beta)+S_{2}(2 \pi \alpha) S_{1}(2 \pi \beta) \\
& -C_{3}(2 \pi(\beta-\alpha)) .
\end{aligned}
$$

Proof. Our starting point will be the following elementary identity

$$
\begin{aligned}
-\frac{1}{l m(l+m)}= & \left(\frac{1}{l(l+m)^{2}}+\frac{1}{l^{2}(l+m)}\right)+\left(\frac{1}{m(l+m)^{2}}+\frac{1}{m^{2}(l+m)}\right) \\
& -\left(\frac{1}{l^{2} m}+\frac{1}{l m^{2}}\right) .
\end{aligned}
$$

Notice that

$$
\begin{aligned}
& \sum_{1 \leq l, m} \frac{\cos 2 \pi(l \alpha+m \beta)}{l(l+m)^{2}}+\sum_{1 \leq l, m} \frac{\cos 2 \pi((l+m) \alpha-m \beta)}{l^{2}(l+m)} \\
& =\sum_{1 \leq l, m} \frac{\cos 2 \pi(l(\alpha-\beta)+(l+m) \beta)}{l(l+m)^{2}}+\sum_{1 \leq l, m} \frac{\cos 2 \pi((l+m)(\alpha-\beta)+l \beta)}{l^{2}(l+m)} \\
& =\sum_{1 \leq l, k} \frac{\cos 2 \pi(l(\alpha-\beta)+k \beta)}{l k^{2}}-\sum_{1 \leq k} \frac{\cos 2 \pi(k \alpha)}{k^{3}} .
\end{aligned}
$$

Using the fact that $\cos 2 \pi(l(\alpha-\beta)+k \beta)=\cos (2 \pi l(\alpha-\beta)) \cos (2 \pi k \beta)-$ $\sin (2 \pi l(\alpha-\beta)) \sin (2 \pi k \beta)$, we can rewrite the previous identity as

$$
\begin{aligned}
& \sum_{1 \leq l} \frac{\cos (2 \pi l(\alpha-\beta))}{l} \sum_{1 \leq k} \frac{\cos (2 \pi k \beta)}{k^{2}}-\sum_{1 \leq l} \frac{\sin (2 \pi l(\alpha-\beta))}{l} \sum_{1 \leq k} \frac{\sin (2 \pi k \beta)}{k^{2}} \\
& -\sum_{1 \leq k} \frac{\cos (2 \pi k \alpha)}{k^{3}} \\
= & C_{1}(2 \pi(\alpha-\beta)) C_{2}(2 \pi \beta)-S_{1}(2 \pi(\alpha-\beta)) S_{2}(2 \pi \beta)-C_{3}(2 \pi \alpha) .
\end{aligned}
$$

By exchanging $\beta$ and $\alpha-\beta$ and adding, we obtain

$$
\begin{aligned}
& \sum_{1 \leq l, m} \frac{\cos 2 \pi(l \alpha+m \beta)}{l(l+m)^{2}}+\sum_{1 \leq l, m} \frac{\cos 2 \pi(l \alpha+m \beta)}{l^{2}(l+m)} \\
& +\sum_{1 \leq l, m} \frac{\cos 2 \pi((l+m) \alpha-m \beta)}{l(l+m)^{2}}+\sum_{1 \leq l, m} \frac{\cos 2 \pi((l+m) \alpha-m \beta)}{l^{2}(l+m)} \\
= & C_{1}(2 \pi(\alpha-\beta)) C_{2}(2 \pi \beta)-S_{1}(2 \pi(\alpha-\beta)) S_{2}(2 \pi \beta) \\
& +C_{2}(2 \pi(\alpha-\beta)) C_{1}(2 \pi \beta)-S_{2}(2 \pi(\alpha-\beta)) S_{1}(2 \pi \beta)-2 C_{3}(2 \pi \alpha) .
\end{aligned}
$$


Analogously we obtain

$$
\begin{aligned}
& \sum_{1 \leq l, m} \frac{\cos 2 \pi(l \alpha+m \beta)}{m(l+m)^{2}}+\sum_{1 \leq l, m} \frac{\cos 2 \pi(l \alpha+m \beta)}{m^{2}(l+m)} \\
& +\sum_{1 \leq l, m} \frac{\cos 2 \pi((l+m) \beta-l \alpha)}{m(l+m)^{2}}+\sum_{1 \leq l, m} \frac{\cos 2 \pi((l+m) \beta-l \alpha)}{m^{2}(l+m)} \\
= & C_{1}(2 \pi(\beta-\alpha)) C_{2}(2 \pi \alpha)-S_{1}(2 \pi(\beta-\alpha)) S_{2}(2 \pi \alpha) \\
& +C_{2}(2 \pi(\beta-\alpha)) C_{1}(2 \pi \alpha)-S_{2}(2 \pi(\beta-\alpha)) S_{1}(2 \pi \alpha)-2 C_{3}(2 \pi \beta),
\end{aligned}
$$

and

$$
\begin{aligned}
& \sum_{1 \leq l, m} \frac{\cos 2 \pi((l+m) \beta-l \alpha)}{l(l+m)^{2}}+\sum_{1 \leq l, m} \frac{\cos 2 \pi((l+m) \beta-l \alpha)}{l^{2}(l+m)} \\
& +\sum_{1 \leq l, m} \frac{\cos 2 \pi((l+m) \alpha-m \beta)}{m(l+m)^{2}}+\sum_{1 \leq l, m} \frac{\cos 2 \pi((l+m) \alpha-m \beta)}{m^{2}(l+m)} \\
= & C_{1}(2 \pi \alpha) C_{2}(2 \pi \beta)+S_{1}(2 \pi \alpha) S_{2}(2 \pi \beta) \\
& +C_{2}(2 \pi \alpha) C_{1}(2 \pi \beta)+S_{2}(2 \pi \alpha) S_{1}(2 \pi \beta)-2 C_{3}(2 \pi(\beta-\alpha)) .
\end{aligned}
$$

On the other hand, we have

$$
\begin{aligned}
& \sum_{1 \leq l, m} \frac{\cos 2 \pi(l \alpha+m \beta)}{l^{2} m}+\sum_{1 \leq l, m} \frac{\cos 2 \pi(l \alpha+m \beta)}{l^{2}} \\
& =\sum_{1 \leq l} \frac{\cos (2 \pi l \alpha)}{l^{2}} \sum_{1 \leq m} \frac{\cos (2 \pi m \beta)}{m}-\sum_{1 \leq l} \frac{\sin (2 \pi l \alpha)}{l^{2}} \sum_{1 \leq m} \frac{\sin (2 \pi m \beta)}{m} \\
& +\sum_{1 \leq l} \frac{\cos (2 \pi l \alpha)}{l} \sum_{1 \leq m} \frac{\cos (2 \pi m \beta)}{m^{2}}-\sum_{1 \leq l} \frac{\sin (2 \pi l \alpha)}{l} \sum_{1 \leq m} \frac{\sin (2 \pi m \beta)}{m^{2}} \\
& =C_{2}(2 \pi \alpha) C_{1}(2 \pi \beta)-S_{2}(2 \pi \alpha) S_{1}(2 \pi \beta)+C_{1}(2 \pi \alpha) C_{2}(2 \pi \beta)-S_{1}(2 \pi \alpha) S_{2}(2 \pi \beta) .
\end{aligned}
$$

As before, we can obtain similar identities by exchanging $\beta$ and $\alpha-\beta$ and $\alpha$ and $\beta-\alpha$.

By combining the previous results, we obtain the desired formula:

$$
\begin{aligned}
& -\sum_{1 \leq k, l} \frac{\cos 2 \pi((k+l) \beta-l \alpha)}{k l(k+l)}-\sum_{1 \leq k, m} \frac{\cos 2 \pi((k+m) \alpha-m \beta)}{k m(k+m)} \\
& -\sum_{1 \leq l, m} \frac{\cos 2 \pi(l \alpha+m \beta)}{l m(l+m)} \\
= & 2 S_{1}(2 \pi(\beta-\alpha)) S_{2}(2 \pi \beta)+2 S_{2}(2 \pi(\beta-\alpha)) S_{1}(2 \pi \beta)-2 C_{3}(2 \pi \alpha) \\
& +2 S_{1}(2 \pi(\alpha-\beta)) S_{2}(2 \pi \alpha)+2 S_{2}(2 \pi(\alpha-\beta)) S_{1}(2 \pi \alpha)-2 C_{3}(2 \pi \beta) \\
& +2 S_{1}(2 \pi \alpha) S_{2}(2 \pi \beta)+2 S_{2}(2 \pi \alpha) S_{1}(2 \pi \beta)-2 C_{3}(2 \pi(\beta-\alpha)) .
\end{aligned}
$$


We are now ready to prove Theorem 6

Proof. [Theorem 6] We express $m_{3}(P)$ in terms of the arguments $\alpha_{i}$ :

$$
\begin{aligned}
m_{3}(P) & =\sum_{1 \leq j, k, l \leq n} m\left(1-e^{2 \pi i \alpha_{j}} x, 1-e^{2 \pi i \alpha_{k}} x, 1-e^{2 \pi i \alpha_{l}} x\right) \\
& =\sum_{1 \leq j, k, l \leq n} m\left(1-x, 1-e^{2 \pi i\left(\alpha_{k}-\alpha_{j}\right)} x, 1-e^{2 \pi i\left(\alpha_{l}-\alpha_{j}\right)} x\right) .
\end{aligned}
$$

We now apply Proposition 25

$$
\begin{aligned}
2 m_{3}(P)= & -\sum_{1 \leq j, k, l \leq n}\left(C_{3}\left(2 \pi\left(\alpha_{k}-\alpha_{j}\right)\right)+C_{3}\left(2 \pi\left(\alpha_{l}-\alpha_{k}\right)\right)+C_{3}\left(2 \pi\left(\alpha_{j}-\alpha_{l}\right)\right)\right) \\
& +\sum_{1 \leq j, k, l \leq n}\left(S_{1}\left(2 \pi\left(\alpha_{l}-\alpha_{k}\right)\right) S_{2}\left(2 \pi\left(\alpha_{l}-\alpha_{j}\right)\right)+S_{2}\left(2 \pi\left(\alpha_{l}-\alpha_{k}\right)\right) S_{1}\left(2 \pi\left(\alpha_{l}-\alpha_{j}\right)\right)\right. \\
& +S_{1}\left(2 \pi\left(\alpha_{k}-\alpha_{l}\right)\right) S_{2}\left(2 \pi\left(\alpha_{k}-\alpha_{j}\right)\right)+S_{2}\left(2 \pi\left(\alpha_{k}-\alpha_{l}\right)\right) S_{1}\left(2 \pi\left(\alpha_{k}-\alpha_{j}\right)\right) \\
& \left.+S_{1}\left(2 \pi\left(\alpha_{k}-\alpha_{j}\right)\right) S_{2}\left(2 \pi\left(\alpha_{l}-\alpha_{j}\right)\right)+S_{2}\left(2 \pi\left(\alpha_{k}-\alpha_{j}\right)\right) S_{1}\left(2 \pi\left(\alpha_{l}-\alpha_{j}\right)\right)\right) \\
= & -3 n \sum_{1 \leq k, l \leq n} C_{3}\left(2 \pi\left(\alpha_{l}-\alpha_{k}\right)\right) \\
& +3 \sum_{1 \leq j, k, l \leq n}\left(S_{1}\left(2 \pi\left(\alpha_{l}-\alpha_{k}\right)\right) S_{2}\left(2 \pi\left(\alpha_{l}-\alpha_{j}\right)\right)+S_{2}\left(2 \pi\left(\alpha_{l}-\alpha_{k}\right)\right) S_{1}\left(2 \pi\left(\alpha_{l}-\alpha_{j}\right)\right)\right) \\
= & -3 n \sum_{1 \leq k, l \leq n} C_{3}\left(2 \pi\left(\alpha_{l}-\alpha_{k}\right)\right)+6 \sum_{1 \leq k, l \leq n} S_{2}\left(2 \pi\left(\alpha_{l}-\alpha_{k}\right)\right) \sum_{j=1}^{n} S_{1}\left(2 \pi\left(\alpha_{l}-\alpha_{j}\right)\right) .
\end{aligned}
$$

We will use the following formula

$$
S_{1}(2 \pi \gamma)=\left\{\begin{array}{l}
-\pi\left(\gamma-\frac{1}{2}\right) 0<\gamma<1 \\
-\pi \gamma=0 \quad \gamma=0 \\
-\pi\left(\gamma+\frac{1}{2}\right)-1<\gamma<0
\end{array}\right.
$$

which can be deduced from the fact that $S_{1}(2 \pi \gamma)=\operatorname{Im}\left(-\log \left(1-e^{2 \pi i \gamma}\right)\right)$.

Thus,

$$
-\frac{2}{3} m_{3}(P)=n \sum_{1 \leq k, l \leq n} C_{3}\left(2 \pi\left(\alpha_{l}-\alpha_{k}\right)\right)+2 \pi \sum_{1 \leq k, l \leq n} S_{2}\left(2 \pi\left(\alpha_{l}-\alpha_{k}\right)\right)\left(\sum_{j=1}^{n}\left(\alpha_{l}-\alpha_{j}\right)-\frac{l-1}{2}+\frac{n-l}{2}\right) .
$$

Notice that $\sum_{1 \leq k, l<n} S_{2}\left(2 \pi\left(\alpha_{l}-\alpha_{k}\right)\right)=0$ because $S_{2}\left(2 \pi\left(\alpha_{l}-\alpha_{k}\right)\right)$ cancels with $S_{2}\left(2 \pi\left(\alpha_{k}-\alpha_{l}\right)\right)$. Then

$$
\begin{aligned}
-\frac{2}{3} m_{3}(P) & =n \sum_{1 \leq k, l \leq n} C_{3}\left(2 \pi\left(\alpha_{l}-\alpha_{k}\right)\right)+2 \pi \sum_{1 \leq k, l \leq n} S_{2}\left(2 \pi\left(\alpha_{l}-\alpha_{k}\right)\right)\left(n \alpha_{l}-\sum_{j=1}^{n} \alpha_{j}-\frac{l-1}{2}+\frac{n-l}{2}\right) \\
& =n \sum_{1 \leq k, l \leq n} C_{3}\left(2 \pi\left(\alpha_{l}-\alpha_{k}\right)\right)+2 \pi \sum_{1 \leq k, l \leq n} S_{2}\left(2 \pi\left(\alpha_{l}-\alpha_{k}\right)\right)\left(n \alpha_{l}-l\right) .
\end{aligned}
$$


By exchanging $k$ with $l$ and taking the semi-sum, we obtain

$$
\begin{aligned}
& -\frac{2}{3} m_{3}(P)=n \sum_{1 \leq k, l \leq n} C_{3}\left(2 \pi\left(\alpha_{l}-\alpha_{k}\right)\right)+\pi \sum_{1 \leq k, l \leq n} S_{2}\left(2 \pi\left(\alpha_{l}-\alpha_{k}\right)\right)\left(n\left(\alpha_{l}-\alpha_{k}\right)+k-l\right) \\
& =n^{2} \zeta(3)+2 n \sum_{1 \leq k<l \leq n} C_{3}\left(2 \pi\left(\alpha_{l}-\alpha_{k}\right)\right) \\
& +2 \pi \sum_{1 \leq k<l \leq n} S_{2}\left(2 \pi\left(\alpha_{l}-\alpha_{k}\right)\right)\left(n\left(\alpha_{l}-\alpha_{k}\right)-(l-k)\right) .
\end{aligned}
$$

Theorem 26 Let $(a, b, c)=1$. For integers $d, m$, let $d_{m}=\frac{d}{(d, m)}$ and $m_{d}=$ $\frac{m}{(d, m)}$. Let $n$ be another integer such that $(d, m) \mid n$. Then we denote by $\left[d_{m}^{-1} n\right]_{m_{d}}$ the unique integer between 0 and $m_{d}-1$ such that it is the solution to the equation $d_{m} x \equiv n\left(\bmod m_{d}\right)$. With this notation we have

$$
\begin{gathered}
-2 m\left(x^{a}-1, x^{b}-1, x^{c}-1\right) \\
=a b c\left(\frac{1}{[a, b]^{3}}+\frac{1}{[b, c]^{3}}+\frac{1}{[c, a]^{3}}\right) \zeta(3) \\
-\frac{\pi}{2 c(a, b)} \sum_{\substack{h=1 \\
b_{a} \nmid c h}}^{\infty} \frac{\cot \left(\pi \frac{\left[a_{b}^{-1}\right]_{b_{a}} c h}{b_{a}}\right)}{h^{2}}-\frac{\pi}{2 b(a, c)} \sum_{\substack{h=1 \\
c_{a} \nmid b h}}^{\infty} \frac{\cot \left(\pi \frac{\left[a_{c}^{-1}\right]_{c_{a}} b h}{c_{a}}\right)}{h^{2}} \\
-\frac{\pi}{2 a(b, c)} \sum_{\substack{h=1 \\
c_{b} \nmid a h}}^{\infty} \frac{\cot \left(\pi \frac{\left[b_{c}^{-1}\right]_{c_{b}} a h}{c_{b}}\right)}{h^{2}}-\frac{\pi}{2 c(b, a)} \sum_{\substack{h=1 \\
a_{b} \nmid c h}}^{\infty} \frac{\cot \left(\pi \frac{\left[b_{a}^{-1}\right]_{a_{b}} c h}{a_{b}}\right)}{h^{2}} \\
-\frac{\pi}{2 b(c, a)} \sum_{\substack{h=1 \\
a_{c} \nmid b h}}^{\infty} \frac{\cot \left(\pi \frac{\left[c_{a}^{-1}\right]_{a_{c}} b h}{a_{c}}\right)}{h^{2}}-\frac{\pi}{2 a(c, b)} \sum_{\substack{h=1 \\
b_{c} \nmid a h}}^{\infty} \frac{\cot \left(\pi \frac{\left[c_{b}^{-1}\right]_{b_{c}} a h}{b_{c}}\right)}{h^{2}} .
\end{gathered}
$$

Proof. First notice that the assumption that $(a, b, c)=1$ is not restrictive, since we have that $m\left(x^{a}-1, x^{b}-1, x^{c}-1\right)=m\left(x^{\frac{a}{(a, b, c)}}-1, x^{\frac{b}{(a, b, c)}}-1, x^{\frac{c}{(a, b, c)}}-1\right)$.

By applying the same ideas as in Lemma 10.

$$
\begin{aligned}
2 m\left(x^{a}-1, x^{b}-1, x^{c}-1\right) & =2 \sum_{j=0}^{a-1} \sum_{k=0}^{b-1} \sum_{l=0}^{c-1} m\left(1-e^{2 \pi i j / a} x, 1-e^{2 \pi i k / b} x, 1-e^{2 \pi i l / c} x\right) \\
& =\sum_{j=0}^{a-1} \sum_{k=0}^{b-1} \sum_{l=0}^{c-1} 2 m\left(1-x, 1-e^{2 \pi i k / b-2 \pi i j / a} x, 1-e^{2 \pi i l / c-2 \pi i j / a} x\right) \\
& =\sum_{j=0}^{a-1} \sum_{k=0}^{b-1} \sum_{l=0}^{c-1} S(j, k, l) .
\end{aligned}
$$


By applying Proposition 25, we obtain that each term in the sum is

$$
\begin{aligned}
S(j, k, l):= & S_{2}\left(2 \pi\left(\frac{l}{c}-\frac{j}{a}\right)\right)\left(S_{1}\left(2 \pi\left(\frac{l}{c}-\frac{k}{b}\right)\right)+S_{1}\left(2 \pi\left(\frac{k}{b}-\frac{j}{a}\right)\right)\right) \\
& +S_{2}\left(2 \pi\left(\frac{k}{b}-\frac{l}{c}\right)\right)\left(S_{1}\left(2 \pi\left(\frac{k}{b}-\frac{j}{a}\right)\right)+S_{1}\left(2 \pi\left(\frac{j}{a}-\frac{l}{c}\right)\right)\right) \\
& +S_{2}\left(2 \pi\left(\frac{j}{a}-\frac{k}{b}\right)\right)\left(S_{1}\left(2 \pi\left(\frac{j}{a}-\frac{l}{c}\right)\right)+S_{1}\left(2 \pi\left(\frac{l}{c}-\frac{k}{b}\right)\right)\right) \\
& -C_{3}\left(2 \pi\left(\frac{l}{c}-\frac{j}{a}\right)\right)-C_{3}\left(2 \pi\left(\frac{k}{b}-\frac{l}{c}\right)\right)-C_{3}\left(2 \pi\left(\frac{j}{a}-\frac{k}{b}\right)\right) .
\end{aligned}
$$

We will apply formula (3). First assume that $\frac{l}{c}>\frac{k}{b}>\frac{j}{a}$. Then

$$
\begin{aligned}
S(j, k, l)= & -\pi S_{2}\left(2 \pi\left(\frac{l}{c}-\frac{j}{a}\right)\right)\left(\frac{l}{c}-\frac{j}{a}-1\right) \\
& -\pi S_{2}\left(2 \pi\left(\frac{k}{b}-\frac{l}{c}\right)\right)\left(\frac{k}{b}-\frac{l}{c}\right)-\pi S_{2}\left(2 \pi\left(\frac{j}{a}-\frac{k}{b}\right)\right)\left(\frac{j}{a}-\frac{k}{b}\right) \\
& -C_{3}\left(2 \pi\left(\frac{l}{c}-\frac{j}{a}\right)\right)-C_{3}\left(2 \pi\left(\frac{k}{b}-\frac{l}{c}\right)\right)-C_{3}\left(2 \pi\left(\frac{j}{a}-\frac{k}{b}\right)\right) .
\end{aligned}
$$

Now assume that $\frac{l}{c}>\frac{k}{b}=\frac{j}{a}$ or $\frac{l}{c}=\frac{k}{b}>\frac{j}{a}$. Then

$S(j, k, l)=-2 \pi S_{2}\left(2 \pi\left(\frac{l}{c}-\frac{j}{a}\right)\right)\left(\frac{l}{c}-\frac{j}{a}-\frac{1}{2}\right)-2 C_{3}\left(2 \pi\left(\frac{l}{c}-\frac{j}{a}\right)\right)-\zeta(3)$.

By considering similar analysis for other cases, we finally get

$$
\begin{gathered}
-2 m\left(x^{a}-1, x^{b}-1, x^{c}-1\right) \\
=\sum_{k=0}^{b-1} \sum_{l=0}^{c-1}\left(a C_{3}\left(2 \pi\left(\frac{k}{b}-\frac{l}{c}\right)\right)+\pi S_{2}\left(2 \pi\left(\frac{k}{b}-\frac{l}{c}\right)\right)\left(a\left(\frac{k}{b}-\frac{l}{c}\right)+H_{a}\left(\frac{k}{b}, \frac{l}{c}\right)\right)\right) \\
+\sum_{j=0}^{a-1} \sum_{l=0}^{c-1}\left(b C_{3}\left(2 \pi\left(\frac{l}{c}-\frac{j}{a}\right)\right)+\pi S_{2}\left(2 \pi\left(\frac{l}{c}-\frac{j}{a}\right)\right)\left(b\left(\frac{l}{c}-\frac{j}{a}\right)+H_{b}\left(\frac{l}{c}, \frac{j}{a}\right)\right)\right) \\
+\sum_{j=0}^{a-1} \sum_{k=0}^{b-1}\left(c C_{3}\left(2 \pi\left(\frac{j}{a}-\frac{k}{b}\right)\right)+\pi S_{2}\left(2 \pi\left(\frac{j}{a}-\frac{k}{b}\right)\right)\left(c\left(\frac{j}{a}-\frac{k}{b}\right)+H_{c}\left(\frac{j}{a}, \frac{k}{b}\right)\right)\right),
\end{gathered}
$$

where, $H_{d}\left(\frac{r}{s}, \frac{u}{v}\right)$ for $\frac{r}{s}<\frac{u}{v}$ denotes the number of rational numbers of the form $\frac{m}{d}$ with $m \in \mathbb{Z}$ that belong to the interval $\left[\frac{r}{s}, \frac{u}{v}\right]$ with the following 
conventions: the cases in which $\frac{m}{d}=\frac{r}{s}$ and $\frac{m}{d}=\frac{u}{v}$ are counted with weight $\frac{1}{2}$ instead of 1 , and $H_{d}\left(\frac{u}{v}, \frac{r}{s}\right)=-H_{d}\left(\frac{r}{s}, \frac{u}{v}\right)$. It is not hard to see that

$$
H_{d}\left(\frac{r}{s}, \frac{u}{v}\right)=\frac{\left\lfloor\frac{d u}{v}\right\rfloor+\left\lceil\frac{d u}{v}\right\rceil-\left\lfloor\frac{d r}{s}\right\rfloor-\left\lceil\frac{d r}{s}\right\rceil}{2} .
$$

We will also use the following notation

$$
\{\alpha\}_{2}:=\alpha-\frac{\lfloor\alpha\rfloor+\lceil\alpha\rceil}{2}=\left\{\begin{array}{l}
\alpha-\lfloor\alpha\rfloor-\frac{1}{2} \alpha \notin \mathbb{Z}, \\
0 \quad \alpha \in \mathbb{Z},
\end{array}\right.
$$

whose Fourier series is

$$
\{\alpha\}_{2}=-\frac{1}{\pi} \sum_{h=1}^{\infty} \frac{\sin (2 \pi \alpha h)}{h} .
$$

We first study the terms of (4) with $C_{3}$. In this case we get

$$
\sum_{k=0}^{b-1} \sum_{l=0}^{c-1} C_{3}\left(2 \pi\left(\frac{k}{b}-\frac{l}{c}\right)\right)
$$

$=\sum_{n=1}^{\infty} \sum_{k=0}^{b-1} \sum_{l=0}^{c-1} \frac{\cos \left(2 \pi\left(\frac{k}{b}-\frac{l}{c}\right) n\right)}{n^{3}}$

$=\sum_{n=1}^{\infty} \frac{\sum_{k=0}^{b-1} \cos \left(2 \pi \frac{k n}{b}\right) \sum_{l=0}^{c-1} \cos \left(2 \pi \frac{l n}{c}\right)+\sum_{k=0}^{b-1} \sin \left(2 \pi \frac{k n}{b}\right) \sum_{l=0}^{c-1} \sin \left(2 \pi \frac{l n}{c}\right)}{n^{3}}$

$=\sum_{\substack{n=1 \\ b|n, c| n}}^{\infty} \frac{b c}{n^{3}}=\frac{b c}{[b, c]^{3}} \zeta(3)$.

Here we have used that $\sum_{k=0}^{b-1} \sin \left(2 \pi \frac{k n}{b}\right)=0$ for any $n, \sum_{k=0}^{b-1} \cos \left(2 \pi \frac{k n}{b}\right)=0$ for $b \nmid n$ and $\sum_{k=0}^{b-1} \cos \left(2 \pi \frac{k n}{b}\right)=b$ for $b \mid n$.

Regarding the terms of (4) with $S_{2}$, we obtain,

$$
\begin{aligned}
& \sum_{k=0}^{b-1} \sum_{l=0}^{c-1} S_{2}\left(2 \pi\left(\frac{k}{b}-\frac{l}{c}\right)\right)\left(\left\{\frac{a k}{b}\right\}_{2}-\left\{\frac{a l}{c}\right\}_{2}\right) \\
= & \sum_{n=1}^{\infty} \sum_{k=0}^{b-1} \sum_{l=0}^{c-1} \frac{\sin \left(2 \pi\left(\frac{k}{b}-\frac{l}{c}\right) n\right)\left(\left\{\frac{a k}{b}\right\}_{2}-\left\{\frac{a l}{c}\right\}_{2}\right)}{n^{2}} \\
= & \sum_{n=1}^{\infty} \frac{\sum_{k=0}^{b-1} \sin \left(2 \pi\left(\frac{k}{b}\right) n\right)\left\{\frac{a k}{b}\right\}_{2} \sum_{l=0}^{c-1} \cos \left(2 \pi\left(\frac{l}{c}\right) n\right)-\sum_{k=0}^{b-1} \sin \left(2 \pi\left(\frac{k}{b}\right) n\right) \sum_{l=0}^{c-1} \cos \left(2 \pi\left(\frac{l}{c}\right) n\right)\left\{\frac{a l}{c}\right\}_{2}}{n^{2}} \\
& -\sum_{n=1}^{\infty} \frac{\sum_{k=0}^{b-1} \cos \left(2 \pi\left(\frac{k}{b}\right) n\right)\left\{\frac{a k}{b}\right\}_{2} \sum_{l=0}^{c-1} \sin \left(2 \pi\left(\frac{l}{c}\right) n\right)-\sum_{k=0}^{b-1} \cos \left(2 \pi\left(\frac{k}{b}\right) n\right) \sum_{l=0}^{c-1} \sin \left(2 \pi\left(\frac{l}{c}\right) n\right)\left\{\frac{a l}{c}\right\}_{2}}{n^{2}} \\
= & \sum_{n=1}^{\infty} \frac{\sum_{k=0}^{b-1} \sin \left(2 \pi\left(\frac{k}{b}\right) n\right)\left\{\frac{a k}{b}\right\}_{2} \sum_{l=0}^{c-1} \cos \left(2 \pi\left(\frac{l}{c}\right) n\right)+\sum_{k=0}^{b-1} \cos \left(2 \pi\left(\frac{k}{b}\right) n\right) \sum_{l=0}^{c-1} \sin \left(2 \pi\left(\frac{l}{c}\right) n\right)\left\{\frac{a l}{c}\right\}_{2}}{n^{2}} .
\end{aligned}
$$


We evaluate $\sum_{k=0}^{b-1} \sin \left(2 \pi\left(\frac{k}{b}\right) n\right)\left\{\frac{a k}{b}\right\}_{2}$. If $b \mid n$ we get zero. If not, we apply the Fourier series for $\{\cdot\}_{2}$ and obtain

$$
\begin{aligned}
\sum_{k=0}^{b-1} \sin \left(2 \pi\left(\frac{k}{b}\right) n\right)\left\{\frac{a k}{b}\right\}_{2} & =-\frac{1}{\pi} \sum_{k=0}^{b-1} \sin \left(2 \pi\left(\frac{k}{b}\right) n\right) \sum_{h=1}^{\infty} \frac{\sin \left(2 \pi \frac{a k}{b} h\right)}{h} \\
& =-\frac{1}{\pi} \sum_{h=1}^{\infty} \frac{\sum_{k=0}^{b-1} \sin \left(2 \pi\left(\frac{k}{b}\right) n\right) \sin \left(2 \pi \frac{a k}{b} h\right)}{h} \\
& =-\frac{1}{\pi} \sum_{h=1}^{\infty} \frac{\sum_{k=0}^{b-1}\left(\cos \left(\frac{2 \pi k}{b}(n-a h)\right)-\cos \left(\frac{2 \pi k}{b}(n+a h)\right)\right)}{2 h}
\end{aligned}
$$

The inner finite sum is different from zero only if $b \mid(n-a h)$ or $b \mid(n+a h)$, in other words, $a h \equiv \pm n(\bmod b)$. Notice that this is only possible if $(a, b) \mid n$. Thus, we assume that $n=(a, b) m$. We write this as $h= \pm\left[a_{b}^{-1} m\right]_{b_{a}}+r b_{a}$ where $r$ is an integer that is either nonnegative or positive depending on the sign for the first term. Thus we get

$$
\begin{aligned}
\sum_{\substack{k=0 \\
b \nmid n,(a, b) \mid n}}^{b-1} \sin \left(2 \pi\left(\frac{k}{b}\right) n\right)\left\{\frac{a k}{b}\right\}_{2} & =-\frac{b}{2 \pi}\left(\frac{1}{\left[a_{b}^{-1} m\right]_{b_{a}}}+\sum_{r=1}^{\infty} \frac{1}{r b_{a}+\left[a_{b}^{-1} m\right]_{b_{a}}}-\frac{1}{r b-\left[a_{b}^{-1} m\right]_{b_{a}}}\right) \\
& =-\frac{(a, b)}{2 \pi}\left(\frac{b_{a}}{\left[a_{b}^{-1} m\right]_{b_{a}}}+2 \frac{\left[a_{b}^{-1} m\right]_{b_{a}}}{b_{a}} \sum_{r=1}^{\infty} \frac{1}{\frac{\left[a_{b}^{-1} m\right]_{b_{a}}^{2}}{b_{a}^{2}}-r^{2}}\right) \\
& =-\frac{(a, b)}{2} \cot \left(\pi \frac{\left[a_{b}^{-1} m\right]_{b_{a}}}{b_{a}}\right) .
\end{aligned}
$$

Putting all of the above together for the terms with $S_{2}$, we obtain

$$
\begin{gathered}
\sum_{k=0}^{b-1} \sum_{l=0}^{c-1} S_{2}\left(2 \pi\left(\frac{k}{b}-\frac{l}{c}\right)\right)\left(\left\{\frac{a k}{b}\right\}_{2}-\left\{\frac{a l}{c}\right\}_{2}\right) \\
=-\frac{c}{2(a, b)} \sum_{\substack{m=1 \\
c \mid m, b_{a} \nmid m,}}^{\infty} \frac{\cot \left(\pi \frac{\left[a_{b}^{-1} m\right]_{b_{a}}}{b_{a}}\right)}{m^{2}}-\frac{b}{2(a, c)} \sum_{\substack{m=1 \\
b \mid m, c_{a} \nmid m,}}^{\infty} \frac{\cot \left(\pi \frac{\left[a_{c}^{-1} m\right]_{c_{a}}}{c_{a}}\right)}{m^{2}} .
\end{gathered}
$$

We now write $m=c h$ in the first term and $m=b h$ in the second term. This can be done since $(a, b, c)=1$. Then

$$
\frac{\left[a_{b}^{-1} m\right]_{b_{a}}}{b_{a}}=\frac{\left[a_{b}^{-1}\right]_{b_{a}} c h}{b_{a}}
$$


and analogously in the second term. Thus equation (5) equals

$$
-\frac{1}{2 c(a, b)} \sum_{\substack{h=1 \\ b_{a} \nmid c h}}^{\infty} \frac{\cot \left(\pi \frac{\left[a_{b}^{-1}\right]_{b_{a}} c h}{b_{a}}\right)}{h^{2}}-\frac{1}{2 b(a, c)} \sum_{\substack{h=1 \\ c_{a} \nmid b h}}^{\infty} \frac{\cot \left(\pi \frac{\left[a_{c}^{-1}\right]_{c_{a}} b h}{c_{a}}\right)}{h^{2}} .
$$

Finally, we get

$$
\begin{gathered}
-2 m\left(x^{a}-1, x^{b}-1, x^{c}-1\right) \\
=a b c\left(\frac{1}{[a, b]^{3}}+\frac{1}{[b, c]^{3}}+\frac{1}{[c, a]^{3}}\right) \zeta(3) \\
-\frac{\pi}{2 c(a, b)} \sum_{\substack{h=1 \\
b_{a} \nmid c h}}^{\infty} \frac{\cot \left(\pi \frac{\left[a_{b}^{-1}\right]_{b_{a}} c h}{b_{a}}\right)}{h^{2}}-\frac{\pi}{2 b(a, c)} \sum_{\substack{h=1 \\
c_{a} \nmid b h}}^{\infty} \frac{\cot \left(\pi \frac{\left[a_{c}^{-1}\right]_{c_{a}} b h}{c_{a}}\right)}{h^{2}} \\
-\frac{\pi}{2 a(b, c)} \sum_{\substack{h=1 \\
c_{b} \nmid a h}}^{\infty} \frac{\cot \left(\pi \frac{\left[b_{c}^{-1}\right]_{c_{b}} a h}{c_{b}}\right)}{h^{2}}-\frac{\pi}{2 c(b, a)} \sum_{\substack{h=1 \\
a_{b} \nmid c h}}^{\infty} \frac{\cot \left(\pi \frac{\left[b_{a}^{-1}\right]_{a_{b}} c h}{a_{b}}\right)}{h^{2}} \\
-\frac{\pi}{2 b(c, a)} \sum_{\substack{h=1 \\
a_{c} \nmid b h}}^{\infty} \frac{\cot \left(\pi \frac{\left[c_{a}^{-1}\right]_{a_{c} b h}}{a_{c}}\right)}{h^{2}}-\frac{\pi}{2 a(c, b)} \sum_{\substack{h=1 \\
b_{c} \nmid a h}}^{\infty} \frac{\cot \left(\pi \frac{\left[c_{b}^{-1}\right]_{b_{c} a h}}{b_{c}}\right)}{h^{2}} .
\end{gathered}
$$

This concludes the proof of Theorem 26 .

We can immediately deduce some particular formulae.

Corollary 27 1. For positive integers $a$ and $b$ with $(a, b)=1$,

$$
m\left(x^{a}-1, x^{b}-1, x^{b}-1\right)=-\frac{2+a^{3}}{2 a^{2} b} \zeta(3)+\frac{\pi}{2 b} \sum_{\substack{h=1 \\ a \nmid h}}^{\infty} \frac{\cot \left(\pi \frac{h}{a}\right)}{h^{2}} .
$$

2. For an odd integer $d$, we have

$$
\begin{gathered}
m\left(x-1, x^{4}-1, x^{2 d}-1\right) \\
=-\frac{9+d^{3}}{16 d^{2}} \zeta(3)+\frac{\pi}{16} \sum_{\substack{h=1 \\
d \nmid 2 h}}^{\infty} \frac{\cot \left(\pi \frac{2 h}{d}\right)}{h^{2}}+\frac{\pi}{8} \sum_{\substack{h=1 \\
d \nmid h}}^{\infty} \frac{\cot \left(\pi \frac{(d+1) h}{2 d}\right)}{h^{2}} .
\end{gathered}
$$


Here are some particular cases

$$
\begin{aligned}
m\left(x-1, x^{b}-1, x^{b}-1\right) & =-\frac{3}{2 b} \zeta(3), \\
m\left(x^{2}-1, x^{b}-1, x^{b}-1\right) & =-\frac{5}{4 b} \zeta(3), \\
m\left(x^{3}-1, x^{b}-1, x^{b}-1\right) & =-\frac{29}{18 b} \zeta(3)+\frac{\pi}{2 \sqrt{3} b} L\left(2, \chi_{-3}\right), \\
m\left(x^{4}-1, x^{b}-1, x^{b}-1\right) & =-\frac{33}{16 b} \zeta(3)+\frac{\pi}{2 b} L\left(2, \chi_{-4}\right) .
\end{aligned}
$$

Here $L(s, \chi)$ denotes the Dirichlet $L$-series in the corresponding character $\chi$, i.e., $L(s, \chi)=\sum_{n=1}^{\infty} \frac{\chi(n)}{n^{s}}$.

\section{Limiting values for $m_{k}$}

In [2], Boyd suggests a different point of view for the study of Lehmer's question. He proposes the study of the set

$$
L=\{m(P): P \text { univariate with integer coefficients }\} \subset[0, \infty) .
$$

(Boyd writes this in terms of the Mahler measure $M(P)=e^{m(P)}$ but we will keep everything in terms of the logarithmic Mahler measure for consistency.) The idea is that Lehmer's question can be translated as whether 0 is a limit point of $L$. In fact, as Boyd points out, if 0 is a limit point of $L$, then $L$ is dense in $[0, \infty)$. A negative answer to Lehmer's question yields a much more interesting $L$. Presumably, $L$ is not closed, since $L$ consists of logarithms of algebraic numbers, but $z_{0}=\frac{7}{2 \pi^{2}} \zeta(3)$ is a limit point of $L$ and we do not expect $z_{0}$ to be the logarithm of an algebraic number. If the above is true and if Lehmer's question has a negative answer, then one could ask about other limit points for $L$.

In this section, we proceed to study limits of some sequences in

$$
L_{2 h+1}=\left\{m_{2 h+1}(P): P \text { univariate with integer coefficients }\right\},
$$

with special focus on 0 as a limit point. Namely, we will show that we can obtain certain values (including 0) as limit of sequences $\left\{m_{2 h+1}\left(P_{n}\right)\right\}_{n}$ where $P_{n} \in \mathbb{Z}[x]$.

By a generalization of a result of Boyd and Lawton (Theorem [30), $m_{k}$ of any multivariate polynomial is a limit of a sequence of $m_{k}$ of univariate polynomials. Therefore, the set

$$
L_{2 h+1}^{\#}=\left\{m_{2 h+1}(P): P \text { multivariate with integer coefficients }\right\},
$$

is included in the closure of $L_{2 h+1}$. We will see that Lehmer's question has a positive answer for $m_{2 h+1}$ for $h \geq 1$. Thus, following Boyd, $L_{2 h+1}^{\#}$ can not be a closed set. 
5.1 Limiting values for $m_{3}$

In order to find limit points of $m_{3}$ of certain sequences of polynomials, we will need the following result.

Lemma 28 1. Let $r \in \mathbb{Z}, r \neq 0$ and $p \in \mathbb{Z}$. Then

$$
\lim _{p \rightarrow \infty} \frac{r \pi}{p} \sum_{\substack{h=1 \\ p \nmid r h}}^{\infty} \frac{\cot \left(\pi \frac{r h}{p}\right)}{h^{2}}=\zeta(3) .
$$

2. Let $p \in \mathbb{Z}$ be odd. Then

$$
\lim _{p \rightarrow \infty} \frac{4 \pi}{p} \sum_{\substack{h=1 \\ p \nmid h}}^{\infty} \frac{\cot \left(\pi \frac{(p+1) h}{2 p}\right)}{h^{2}}=\zeta(3) .
$$

Proof. Part 1. Observe that $\cot (x)<\frac{1}{x}$ for $0<x<\pi$. Thus, for $0<h<\frac{p}{r}$, we can write

$$
\cot \left(\pi \frac{r h}{p}\right)<\frac{p}{r h \pi}
$$

Moreover, for $p \nmid h$, we have that

$$
\left|\cot \left(\pi \frac{r h}{p}\right)\right|<\frac{p}{\pi}
$$

Thus,

$$
\begin{aligned}
\frac{r \pi}{p} \sum_{\substack{h=1 \\
p \nmid r h}}^{\infty} \frac{\cot \left(\pi \frac{r h}{p}\right)}{h^{2}} & =\frac{r \pi}{p} \sum_{1 \leq h<\frac{p}{r}} \frac{\cot \left(\pi \frac{r h}{p}\right)}{h^{2}}+\frac{r \pi}{p} \sum_{\substack{p \\
r \\
p \nmid h h}} \frac{\cot \left(\pi \frac{r h}{p}\right)}{h^{2}} \\
& <\sum_{1 \leq h<\frac{p}{r}} \frac{1}{h^{3}}+r \sum_{\frac{p}{r}<h} \frac{1}{h^{2}}
\end{aligned}
$$

On the other hand, $\lim _{x \rightarrow 0} x \cot (x)=1$. Given $\epsilon>0$, take $p$ large enough such that

$$
\cot \left(\pi \frac{r h}{p}\right) \geq \frac{p}{r h \pi}(1-\epsilon)
$$

for any $0<h<\sqrt{p}$. Let $H=\lfloor\sqrt{p}\rfloor$. Then

$$
\begin{aligned}
\frac{r \pi}{p} \sum_{\substack{h=1 \\
p \nmid r h}}^{\infty} \frac{\cot \left(\pi \frac{r h}{p}\right)}{h^{2}} & =\frac{r \pi}{p} \sum_{\substack{h=1 \\
p \nmid r h}}^{H} \frac{\cot \left(\pi \frac{r h}{p}\right)}{h^{2}}+\frac{r \pi}{p} \sum_{\substack{h=H \\
p \nmid r h}}^{\infty} \frac{\cot \left(\pi \frac{r h}{p}\right)}{h^{2}} \\
& \geq(1-\epsilon) \sum_{h=1}^{H} \frac{1}{h^{3}}-\frac{r \pi}{p} \sum_{\substack{h=H \\
p \nmid r h}}^{\infty} \frac{\left|\cot \left(\pi \frac{r h}{p}\right)\right|}{h^{2}} \\
& \geq(1-\epsilon) \sum_{h=1}^{H} \frac{1}{h^{3}}-r \sum_{h=H}^{\infty} \frac{1}{h^{2}}
\end{aligned}
$$


Taking the limit when $p \rightarrow \infty$, we conclude that

$$
\lim _{p \rightarrow \infty} \frac{r \pi}{p} \sum_{\substack{h=1 \\ p \nmid r h}}^{\infty} \frac{\cot \left(\pi \frac{r h}{p}\right)}{h^{2}}=\zeta(3) .
$$

Part 2.

$$
\frac{4 \pi}{p} \sum_{\substack{h=1 \\ p \nmid h}}^{\infty} \frac{\cot \left(\pi \frac{(p+1) h}{2 p}\right)}{h^{2}}=\frac{4 \pi}{p} \sum_{\substack{h=1 \\ 2 \mid h, p \nmid h}}^{\infty} \frac{\cot \left(\pi \frac{(p+1) h}{2 p}\right)}{h^{2}}+\frac{4 \pi}{p} \sum_{\substack{h=1 \\ 2 \nmid h, p \nmid h}}^{\infty} \frac{\cot \left(\pi \frac{(p+1) h}{2 p}\right)}{h^{2}} .
$$

For the first term, we let $h=2 j$. For the second term, we observe that, for $0<h<p$,

$$
\cot \left(\pi \frac{(p+1) h}{2 p}\right)=\cot \left(\frac{\pi}{2}+\frac{h \pi}{2 p}\right)=\cot \left(\pi \frac{p+h}{2 p}\right)<\frac{2 p}{\pi(p+h)},
$$

and for $p \nmid h$,

$$
\left|\cot \left(\pi \frac{(p+1) h}{2 p}\right)\right|<\frac{2 p}{\pi} .
$$

Thus, equation (6) equals

$$
\begin{aligned}
& \frac{\pi}{p} \sum_{\substack{j=1 \\
p \nmid j}}^{\infty} \frac{\cot \left(\pi \frac{(p+1) j}{p}\right)}{j^{2}}+\frac{4 \pi}{p} \sum_{1 \leq h<p} \frac{\cot \left(\pi \frac{(p+1) h}{2 p}\right)}{h^{2}}+\frac{4 \pi}{p} \sum_{\substack{p<h \\
p \nmid h}}^{\infty} \frac{\cot \left(\pi \frac{(p+1) h}{2 p}\right)}{h^{2}} \\
\leq & \frac{\pi}{p} \sum_{\substack{j=1 \\
p \nmid j}}^{\infty} \frac{\cot \left(\pi \frac{j}{p}\right)}{j^{2}}+8 \sum_{1 \leq h<p} \frac{1}{(p+h) h^{2}}+8 \sum_{\substack{p<h \\
p \nmid h}}^{\infty} \frac{1}{h^{2}} \\
\leq & \frac{\pi}{p} \sum_{\substack{j=1 \\
p \nmid j}}^{\infty} \frac{\cot \left(\pi \frac{j}{p}\right)}{j^{2}}+\frac{8}{p} \zeta(2)+8 \sum_{\substack{p<h \\
p \nmid h}}^{\infty} \frac{1}{h^{2}} .
\end{aligned}
$$

Similarly, we can write

$$
\frac{4 \pi}{p} \sum_{\substack{h=1 \\ p \nmid h}}^{\infty} \frac{\cot \left(\pi \frac{(p+1) h}{2 p}\right)}{h^{2}} \geq \frac{\pi}{p} \sum_{\substack{j=1 \\ p \nmid j}}^{\infty} \frac{\cot \left(\pi \frac{j}{p}\right)}{j^{2}}-\frac{8}{p} \zeta(2)-8 \sum_{\substack{p<h \\ p \nmid h}}^{\infty} \frac{1}{h^{2}} .
$$

By taking the limit when $p \rightarrow \infty$ and using Part 1, we conclude the proof.

We will now compute $m_{3}$ for some sequences of polynomials and take their limits. This process will provide us with limit points for the values of $m_{3}$ as well as infinitely many polynomials $P$ with positive and negative values of $m_{3}(P)$. 
1. Consider the family of polynomials $\frac{x^{p}-1}{x-1}$. From part 1 of Corollary 27 we have that

$$
\begin{aligned}
m_{3}\left(\frac{x^{p}-1}{x-1}\right) & =m_{3}\left(x^{p}-1\right)-m_{3}(x-1)+3 m\left(x^{p}-1, x-1, x-1\right) \\
& -3 m\left(x^{p}-1, x^{p}-1, x-1\right) \\
& =3\left(-\frac{2+p^{3}}{2 p^{2}} \zeta(3)+\frac{\pi}{2} \sum_{\substack{h=1 \\
p \nmid h}}^{\infty} \frac{\cot \left(\pi \frac{h}{p}\right)}{h^{2}}+\frac{3}{2 p} \zeta(3)\right) \\
& =\frac{9 p-6-3 p^{3}}{2 p^{2}} \zeta(3)+\frac{3 \pi}{2} \sum_{\substack{h=1 \\
p \nmid h}}^{\infty} \frac{\cot \left(\pi \frac{h}{p}\right)}{h^{2}} .
\end{aligned}
$$

Thus,

$$
\begin{aligned}
\lim _{p \rightarrow \infty} m_{3}\left(\frac{x^{p}-1}{x-1}\right) & =\lim _{p \rightarrow \infty}\left(\frac{9 p-6-3 p^{3}}{2 p^{2}} \zeta(3)+\frac{3 \pi}{2} \sum_{\substack{h=1 \\
p \nmid h}}^{\infty} \frac{\cot \left(\pi \frac{h}{p}\right)}{h^{2}}\right) \\
& =\lim _{p \rightarrow \infty}\left(\frac{9 p-6-3 p^{3}}{2 p^{2}} \zeta(3)+\frac{3 p}{2} \zeta(3)\right) \\
& =0 .
\end{aligned}
$$

Thus, 0 seems to be a limit point for $L_{3}$.

2. Now, let us focus on the case of $\left(x^{p}-1\right)(x-1)$. Again, we apply part 1 of Corollary 27, in order to obtain

$$
\begin{aligned}
m_{3}\left(\left(x^{p}-1\right)(x-1)\right) & =m_{3}\left(x^{p}-1\right)+m_{3}(x-1)+3 m\left(x^{p}-1, x-1, x-1\right) \\
& +3 m\left(x^{p}-1, x^{p}-1, x-1\right) \\
& =3\left(-\zeta(3)-\frac{2+p^{3}}{2 p^{2}} \zeta(3)+\frac{\pi}{2} \sum_{\substack{h=1 \\
p \nmid h}}^{\infty} \frac{\cot \left(\pi \frac{h}{p}\right)}{h^{2}}-\frac{3}{2 p} \zeta(3)\right) \\
& =\frac{-6 p^{2}-9 p-6-3 p^{3}}{2 p^{2}} \zeta(3)+\frac{3 \pi}{2} \sum_{\substack{h=1 \\
p \nmid h}}^{\infty} \frac{\cot \left(\pi \frac{h}{p}\right)}{h^{2}} .
\end{aligned}
$$

Thus,

$$
\begin{aligned}
\lim _{p \rightarrow \infty} m_{3}\left(\left(x^{p}-1\right)(x-1)\right) & =\lim _{p \rightarrow \infty}\left(\frac{-6 p^{2}-9 p-6-3 p^{3}}{2 p^{2}} \zeta(3)+\frac{3 \pi}{2} \sum_{\substack{h=1 \\
p \nmid h}}^{\infty} \frac{\cot \left(\pi \frac{h}{p}\right)}{h^{2}}\right) \\
& =\lim _{p \rightarrow \infty}\left(\frac{-6 p^{2}-9 p-6-3 p^{3}}{2 p^{2}} \zeta(3)+\frac{3 p}{2} \zeta(3)\right) \\
& =-3 \zeta(3) .
\end{aligned}
$$


Thus, $-3 \zeta(3)$ seems to be a limit point for $L_{3}$. In addition, we obtain infinitely many polynomials $P$ such that $m_{3}(P)<0$.

3. We now look at the case $a=1, b=4$ and $c=2 d$ with $d$ odd. Applying part 2 of Corollary 27 and observing that for an odd integer $d,\left[2^{-1}\right]_{d}=\frac{d+1}{2}$, we get

$$
\begin{aligned}
& m_{3}\left(\frac{\left(x^{4}-1\right)\left(x^{2 d}-1\right)}{(x-1)^{2}}\right)=m_{3}\left(x^{4}-1\right)+m_{3}\left(x^{2 d}-1\right)-8 m_{3}(x-1) \\
& +3 m\left(x^{4}-1, x^{2 d}-1, x^{2 d}-1\right) \\
& +3 m\left(x^{4}-1, x^{4}-1, x^{2 d}-1\right) \\
& +12 m\left(x^{4}-1, x-1, x-1\right) \\
& -6 m\left(x^{4}-1, x^{4}-1, x-1\right) \\
& +12 m\left(x^{2 d}-1, x-1, x-1\right) \\
& -6 m\left(x^{2 d}-1, x^{2 d}-1, x-1\right) \\
& -12 m\left(x^{4}-1, x^{2 d}-1, x-1\right) \\
& =-\frac{3}{2} \zeta(3)-\frac{3}{2} \zeta(3)+12 \zeta(3) \\
& -\frac{15}{4 d} \zeta(3)-\frac{6+3 d^{3}}{4 d^{2}} \zeta(3)+\frac{3 \pi}{4} \sum_{\substack{h=1 \\
d \nmid h}}^{\infty} \frac{\cot \left(\pi \frac{h}{d}\right)}{h^{2}} \\
& -\frac{99}{4} \zeta(3)+6 \pi L\left(2, \chi_{-4}\right)+\frac{9}{4} \zeta(3) \\
& -\frac{3+12 d^{3}}{d^{2}} \zeta(3)+6 \pi \sum_{\substack{h=1 \\
2 d \nmid h}}^{\infty} \frac{\cot \left(\pi \frac{h}{2 d}\right)}{h^{2}}+\frac{9}{2 d} \zeta(3) \\
& +\frac{27+3 d^{3}}{4 d^{2}} \zeta(3)-\frac{3 \pi}{4} \sum_{\substack{h=1 \\
d \nmid 2 h}}^{\infty} \frac{\cot \left(\pi \frac{2 h}{d}\right)}{h^{2}} \\
& -\frac{3 \pi}{2} \sum_{\substack{h=1 \\
d \nmid h}}^{\infty} \frac{\cot \left(\pi \frac{(d+1) h}{2 d}\right)}{h^{2}} \\
& =\frac{9+3 d-54 d^{2}-48 d^{3}}{4 d^{2}} \zeta(3)+6 \pi L\left(2, \chi_{-4}\right) \\
& +\frac{3 \pi}{4} \sum_{\substack{h=1 \\
d \nmid h}}^{\infty} \frac{\cot \left(\pi \frac{h}{d}\right)}{h^{2}}+6 \pi \sum_{\substack{h=1 \\
2 d \nmid h}}^{\infty} \frac{\cot \left(\pi \frac{h}{2 d}\right)}{h^{2}} \\
& -\frac{3 \pi}{4} \sum_{\substack{h=1 \\
d \nmid 2 h}}^{\infty} \frac{\cot \left(\pi \frac{2 h}{d}\right)}{h^{2}}-\frac{3 \pi}{2} \sum_{\substack{h=1 \\
d \nmid h}}^{\infty} \frac{\cot \left(\pi \frac{(d+1) h}{2 d}\right)}{h^{2}} .
\end{aligned}
$$


Thus

$$
\begin{aligned}
\lim _{d \rightarrow \infty} m_{3}\left(\frac{\left(x^{4}-1\right)\left(x^{2 d}-1\right)}{(x-1)^{2}}\right)= & \lim _{d \rightarrow \infty}\left(\frac{9+3 d-54 d^{2}-48 d^{3}}{4 d^{2}} \zeta(3)\right. \\
& +6 \pi L(2, \chi-4)+\frac{3 \pi}{4} \sum_{h=1}^{\infty} \frac{\cot \left(\pi \frac{h}{d}\right)}{h^{2}} \\
& +6 \pi \sum_{\substack{h=1 \\
2 d \nmid h}}^{\infty} \frac{\cot \left(\pi \frac{h}{2 d}\right)}{h^{2}}-\frac{3 \pi}{4} \sum_{h=1}^{\infty} \frac{\cot \left(\pi \frac{2 h}{d}\right)}{h^{2}} \\
& \left.-\frac{3 \pi}{2} \sum_{h=1}^{\infty} \frac{\cot \left(\pi \frac{(d+1) h}{2 d}\right)}{h^{2}}\right) \\
= & \lim _{d \rightarrow \infty}\left(\frac{9+3 d-54 d^{2}-48 d^{3}}{4 d^{2}} \zeta(3)\right. \\
& +6 \pi L(2, \chi-4)+\frac{3 d}{4} \zeta(3) \\
& \left.+12 d \zeta(3)-\frac{3 d}{8} \zeta(3)-\frac{3 d}{8} \zeta(3)\right) \\
= & 6 \pi L(2, \chi-4)-\frac{27}{2} \zeta(3) \cong 1.0377764969 \ldots
\end{aligned}
$$

Thus, $6 \pi L\left(2, \chi_{-4}\right)-\frac{27}{2} \zeta(3)$ seems to be a limit point for $L_{3}$. In addition, we obtain infinitely many polynomials $P$ such that $m_{3}(P)>0$.

4. It is not generally hard to find positive limit points for $m_{3}(P)$, for example, one can take the sequence $\left(x^{n}+3\right)(x+3)$. It is clear that $m_{3}\left(\left(x^{n}+3\right)(x+\right.$ 3)) $\geq \log ^{3} 4>0$.

\subsection{Limit values for higher Mahler measures}

Analogously to the Mahler measure for one variable, the Mahler measure of a non-zero multi-variable polynomial $P\left(x_{1}, \ldots, x_{n}\right) \in \mathbb{C}\left[x_{1}, \ldots, x_{n}\right]$ can be defined as

$$
m(P):=\frac{1}{(2 \pi i)^{n}} \int_{\left|x_{1}\right|=1} \ldots \int_{\left|x_{n}\right|=1} \log \left|P\left(x_{1}, \ldots, x_{n}\right)\right| \frac{d x_{1}}{x_{1}} \ldots \frac{d x_{n}}{x_{n}} .
$$

This generalization can be extended to the multiple (and higher) Mahler measure. Let $P_{1}, \ldots, P_{l} \in \mathbb{C}\left[x_{1}, \ldots, x_{n}\right]$ be nonzero polynomials. Then, we define $m\left(P_{1}, \ldots, P_{l}\right)$ as

$\frac{1}{(2 \pi i)^{n}} \int_{\left|x_{1}\right|=1} \ldots \int_{\left|x_{n}\right|=1} \log \left|P_{1}\left(x_{1}, \ldots, x_{n}\right)\right| \ldots \log \left|P_{l}\left(x_{1}, \ldots, x_{n}\right)\right| \frac{d x_{1}}{x_{1}} \ldots \frac{d x_{n}}{x_{n}}$.

Boyd [3] conjectured the following important statement, which was completely proved by Lawton 8 . 
Theorem 29 Let $P\left(x_{1}, \ldots, x_{n}\right) \in \mathbb{C}\left[x_{1}, \ldots, x_{n}\right]$ and $\mathbf{r}=\left(r_{1}, \ldots, r_{n}\right), r_{i} \in$ $\mathbb{Z}_{>0}$. Define $P_{\mathbf{r}}(x)$ as

$$
P_{\mathbf{r}}(x)=P\left(x^{r_{1}}, \ldots, x^{r_{n}}\right)
$$

and let

$$
q(\mathbf{r})=\min \left\{H(\mathbf{s}): \mathbf{s}=\left(s_{1}, \ldots, s_{n}\right) \in \mathbb{Z}^{n}, \mathbf{s} \neq(0, \ldots, 0), \sum_{j=1}^{n} s_{j} r_{j}=0\right\},
$$

where $H(\mathbf{s})=\max \left\{\left|s_{j}\right|: 1 \leq j \leq n\right\}$. Then

$$
\lim _{q(\mathbf{r}) \rightarrow \infty} m\left(P_{\mathbf{r}}\right)=m(P) \text {. }
$$

It is a simple exercise to generalize the techniques of Lawton to prove an analogous result for multiple Mahler measures. That is, under the same conditions as above, one can show

Theorem 30 Let $P_{1}, \ldots, P_{l} \in \mathbb{C}\left[x_{1}, \ldots, x_{n}\right]$, and $\mathbf{r}$ as before. Then

$$
\lim _{q(\mathbf{r}) \rightarrow \infty} m\left(P_{1 \mathbf{r}}, \ldots, P_{l \mathbf{r}}\right)=m\left(P_{1}, \ldots, P_{l}\right) .
$$

As an immediate application of Theorem 30, we get that for any $a \geq 1$,

$$
\lim _{p \rightarrow \infty} m\left(x^{a}-1, x^{p}-1, x^{p}-1\right)=m(x-1) m_{2}(y-1)=0
$$

and

$$
\lim _{p \rightarrow \infty} m\left(x^{a}-1, x^{a}-1, x^{p}-1\right)=m_{2}(x-1) m(y-1)=0 .
$$

Thus, the limits from Section 5.1 follow from this. An advantage of Theorem 30 over the techniques in Section 5.1 is that it gives us the limits of $m_{k}$ of these sequences for all values of $k$. For example, we immediately obtain that

$$
\lim _{n \rightarrow \infty} m_{2 h+1}\left(\frac{x^{n}-1}{x-1}\right)=0
$$

We will prove in the next subsection that the above sequence (for $h>0$ fixed) is nonconstant. While 0 is a limit point of $m_{2 h+1}$, what can be said about positive and negative values? As in the case of $m_{3}$, it is not hard to see that $m_{k}(x+3) \geq \log ^{k} 2>0$. Using Theorem 30 we can see that the sequence $m_{2 h+1}\left(\left(x^{n}+3\right)(x+3)\right)$ has a positive limit. As for negative limits, the sequence $m_{2 h+1}\left(\left(x^{n}-1\right)(x-1)\right)$ provides a good example. To see this, we apply the following result from [6] (Theorem 3):

Theorem 31 For $l \in \mathbb{Z}_{\geq 1}$,

$$
m_{l}(x-1)=\sum_{b_{1}+\cdots+b_{j}=l, b_{i} \geq 2} \frac{(-1)^{l} l !}{2^{2 j}} \zeta\left(b_{1}, \ldots, b_{j}\right),
$$

where

$$
\zeta\left(b_{1}, \ldots, b_{j}\right)=\sum_{1 \leq p_{1}<\cdots<p_{j}} \frac{1}{p_{1}^{b_{1}} \ldots p_{j}^{b_{j}}} .
$$


From Theorem 30, we get that

$$
\begin{gathered}
\lim _{n \rightarrow \infty} m_{2 h+1}\left(\left(x^{n}-1\right)(x-1)\right)=m_{2 h+1}((y-1)(x-1)) \\
=\sum_{i=0}^{2 h+1}\left(\begin{array}{c}
2 h+1 \\
i
\end{array}\right) m_{i}(y-1) m_{2 h+1-i}(x-1) .
\end{gathered}
$$

Moreover, Theorem 31 tells us that $m_{l}(x-1)<0$ for odd $l$ and $m_{l}(x-1)>0$ for even $l$, that is, each term on the right hand side of the above equation is negative. Thus, $m_{2 h+1}\left(\left(x^{n}-1\right)(x-1)\right)$ has a negative limit.

On a different note, observe that $\frac{\pi^{2}}{12}$ is a limiting value for $m_{2}$, since, by Theorem 19 (iv) in [6], we have that $m_{2}(x+y+2)=\frac{\pi^{2}}{12}$. Thus

$$
\lim _{n \rightarrow \infty} m_{2}\left(x^{n}+x+2\right)=\frac{\pi^{2}}{12}
$$

5.3 A proof that certain sequences are nonconstant

As usual, Theorem 30 does not say anything about the sequence of values $m_{2 h+1}\left(\frac{x^{n}-1}{x-1}\right)$, which in principle could be constant (and therefore, identically zero). This is precisely the case with $h=0$.

Fortunately, we have the following result.

Theorem 32 Let $h \geq 1$ fixed and $P_{n}(x)=\frac{x^{n}-1}{x-1}$. Then the sequence $m_{2 h+1}\left(P_{n}\right)$ is nonconstant.

The idea of this proof was provided to us by Kannan Soundararajan. We will need some auxiliary results first.

Lemma 33 Let $\alpha, m \in \mathbb{Z}$ with $m$ positive. Let

$$
T_{m}(\alpha):=\sum_{\substack{\ell_{1}, \ldots, \ell_{m} \in \mathbb{Z} \neq 0 \\ \ell_{1}+\cdots+\ell_{m}=\alpha}} \frac{1}{\left|\ell_{1}\right| \ldots\left|\ell_{m}\right|}
$$

Then, for $\alpha \neq 0$,

$$
T_{m}(\alpha)=\frac{2^{m-1} m \log ^{m-1}|\alpha|}{|\alpha|}\left(1+O\left(\log ^{-2}|\alpha|\right)\right)
$$


Proof. First notice that $T_{m}(\alpha)=T_{m}(-\alpha)$, so we can assume that $\alpha$ is positive.

By multiplying and dividing by $\ell_{1}+\cdots+\ell_{m}=\alpha$, we obtain that

$$
\begin{aligned}
& T_{m}(\alpha)=\frac{1}{\alpha} \sum_{\substack{\ell_{1}, \ldots, \ell_{m} \in \mathbb{Z} \neq 0 \\
\ell_{1}+\cdots+\ell_{m}=\alpha}} \frac{\ell_{1}+\cdots+\ell_{m}}{\left|\ell_{1}\right| \ldots\left|\ell_{m}\right|}=\frac{1}{\alpha} \sum_{j=1}^{m} \sum_{\ell_{j} \in \mathbb{Z}_{\neq 0}} \operatorname{sign}\left(\ell_{j}\right) T_{m-1}\left(\alpha-\ell_{j}\right) \\
& =\frac{m}{\alpha} \sum_{\ell \in \mathbb{Z}_{\neq 0}} \operatorname{sign}(\ell) T_{m-1}(\alpha-\ell) \\
& =\frac{m}{\alpha}\left(-\sum_{\ell=-\infty}^{-1} T_{m-1}(\alpha-\ell)+\sum_{\ell=1}^{\alpha} T_{m-1}(\alpha-\ell)+\sum_{\ell=\alpha+1}^{2 \alpha} T_{m-1}(\alpha-\ell)+\sum_{\ell=2 \alpha+1}^{\infty} T_{m-1}(\alpha-\ell)\right) \\
& =\frac{2 m}{\alpha} \sum_{j=0}^{\alpha} T_{m-1}(j)-\frac{m}{\alpha}\left(T_{m-1}(0)+T_{m-1}(\alpha)\right) \text {. }
\end{aligned}
$$

Now observe that $T_{1}(\alpha)=\frac{1}{|\alpha|}$ for $\alpha \neq 0$. We proceed by induction. Assume that the statement is true for $m$. Then

$$
\begin{aligned}
T_{m+1}(\alpha) & =\frac{2(m+1)}{\alpha} \sum_{j=0}^{\alpha} T_{m}(j)-\frac{m+1}{\alpha}\left(T_{m}(0)+T_{m}(\alpha)\right) \\
& =\frac{2(m+1)}{\alpha} \sum_{j=1}^{\alpha} \frac{2^{m-1} m \log ^{m-1} j}{j}\left(1+O\left(\log ^{-2} j\right)\right)-\frac{m+1}{\alpha} \frac{2^{m-1} m \log ^{m-1} \alpha}{\alpha}\left(1+O\left(\log ^{-2} \alpha\right)\right)
\end{aligned}
$$

We now replace the above sum with the integral of $\frac{\log ^{m-1} x}{x}$ (with exponent $m-3$ for the error term). This replacement introduces another error term of $O\left(\frac{\log ^{m-1} \alpha}{\alpha}\right)$. We deduce that

$$
\begin{aligned}
T_{m+1}(\alpha) & =\frac{2^{m}(m+1)}{\alpha}\left(\log ^{m} \alpha+O\left(\frac{\log ^{m-1} \alpha}{\alpha}\right)\right)+O\left(\frac{\log ^{m-2} \alpha}{\alpha}\right)-\frac{2^{m-1} m(m+1) \log ^{m-1} \alpha}{\alpha^{2}}\left(1+O\left(\log ^{-2} \alpha\right)\right) \\
& =\frac{2^{m}(m+1)}{\alpha} \log ^{m} \alpha\left(1+O\left(\log ^{-2} \alpha\right)\right)
\end{aligned}
$$

Proposition 34 Let $j, k \in \mathbb{Z}_{\geq 1}$. There is a positive constant $C(j, k)$ such that

$$
U_{j, k}^{(n)}:=\sum_{\substack{\ell_{1}, \ldots, \ell_{j+k} \in \mathbb{Z} \neq 0 \\ \ell_{1}+\cdots+\ell_{j}+n \ell_{j+1}+\cdots+n \ell_{j+k}=0}} \frac{1}{\left|\ell_{1}\right| \ldots\left|\ell_{j+k}\right|}=C(j, k) \frac{\log ^{j-1} n}{n}\left(1+O\left(\log ^{-1} n\right)\right) .
$$


Proof. We have that

$$
\begin{aligned}
U_{j, k}^{(n)} & =\sum_{\alpha \in \mathbb{Z}} T_{j}(n \alpha) T_{k}(-\alpha) \\
& =2 \sum_{\alpha=1}^{\infty} \frac{2^{j-1} j \log ^{j-1}|n \alpha|}{|n \alpha|}\left(1+O\left(\log ^{-2}|n \alpha|\right)\right) T_{k}(\alpha) .
\end{aligned}
$$

We only need to study the behavior when $n$ goes to infinity. Therefore, we do not need to have $\alpha$ in the error term. We write $\log ^{j-1}|n \alpha|=\log ^{j-1}|n|+$ $O\left(\log ^{j-2}|n|\right)$ and we obtain

$$
U_{j, k}^{(n)}=\frac{2^{j} j \log ^{j-1} n}{n} \sum_{\alpha=1}^{\infty} \frac{T_{k}(\alpha)}{\alpha}\left(1+O\left(\log ^{-1} n\right)\right) .
$$

Notice that $T_{k}(\alpha)>0$ by construction, and so is $C(j, k)$.

Proof.[Theorem 32 By writing the integral and using Fourier expansions, we obtain

$$
\begin{aligned}
m_{2 h+1}\left(P_{n}\right) & =\int_{0}^{1} \log ^{2 h+1}\left|\frac{e^{2 \pi i n \theta}-1}{e^{2 \pi i \theta}-1}\right| d \theta \\
& =\sum_{j=0}^{2 h+1}\left(\begin{array}{c}
2 h+1 \\
j
\end{array}\right)(-1)^{j} \int_{0}^{1} \log ^{j}\left|e^{2 \pi i \theta}-1\right| \log ^{2 h+1-j}\left|e^{2 \pi i n \theta}-1\right| d \theta \\
& =\sum_{j=0}^{2 h+1}\left(\begin{array}{c}
2 h+1 \\
j
\end{array}\right)(-1)^{j} \int_{0}^{1}\left(-\frac{1}{2} \sum_{\ell_{1} \in \mathbb{Z}_{\neq 0}} \frac{e^{2 \pi i \ell_{1} \theta}}{\left|\ell_{1}\right|}\right)^{j}\left(-\frac{1}{2} \sum_{\ell_{2} \in \mathbb{Z}_{\neq 0}} \frac{e^{2 \pi i n \ell_{2} \theta}}{\left|\ell_{2}\right|}\right)^{2 h+1-j} d \theta \\
& =\sum_{j=0}^{2 h+1}\left(\begin{array}{c}
2 h+1 \\
j
\end{array}\right) \frac{(-1)^{j+1}}{2^{2 h+1}} U_{j, 2 h+1-j}^{(n)} .
\end{aligned}
$$

By Proposition 34, the term with the highest weight in $n$ is for $j=2 h$. Notice that the condition $h \geq 1$ is necessary because otherwise we obtain a formula that does not depend on $n$. Thus, we have

$$
m_{2 h+1}\left(P_{n}\right)=-\frac{(2 h+1)}{2^{2 h+1}} C(2 h, 1) \frac{\log ^{2 h-1} n}{n}\left(1+O\left(\log ^{-1} n\right)\right) .
$$

Therfore, $m_{2 h+1}\left(P_{n}\right)$ behaves like a nonzero constant times $\frac{\log ^{2 h-1} n}{n}$ when $n$ goes to infinity. This implies that the sequence can not be identically zero.

The discussion in this section proves Theorem 7. 
6 Discussion on the values of $m_{k}(P)$

We will once again focus our attention on the set

$$
L_{k}=\left\{m_{k}(P): P \text { univariate with integer coefficients }\right\} .
$$

For $k=2$, we have

$$
L_{2}=\left\{m_{2}(P): P \text { univariate with integer coefficients }\right\} \subset\left[\frac{\pi^{2}}{48}, \infty\right) .
$$

In this context, the first noticeable difference between $m(P)$ and $m_{2}(P)$ is that the cyclotomic polynomials are interesting in terms of $m_{2}(P)$. We have explored this phenomenon in this note. Many questions remain, however, and in particular, the question of what happens with the reciprocal noncyclotomic polynomials -the ones that are interesting in the case of the classical Mahler measure- is presumably as interesting and difficult as in the case of the classical Mahler measure. In particular, equation (1) and Proposition 18 suggest that a natural object to study is $m_{2}(P)-m(P)^{2}$.

The following table records the noncyclotomic polynomials of degree less or equal than 14 with $m(P)<0.25$. The data has been obtained from the generator in Mossinghoff's website [1]. We observe that the smallest polynomial (in the table) in terms of $m_{2}(P)$ is not the degree-10 polynomial of Lehmer, but $x^{10}+x^{9}-x^{5}+x+1$. In fact, all the polynomials in the table have $m_{2}(P)$ smaller than Lehmer's polynomial. This result comes from the fact that the term $m(P)^{2}$ in equation (11) seems considerably smaller than the other terms, and therefore, the contribution of $m(P)$ to the value of $m_{2}(P)$ is relatively small for polynomials of small $m(P)$. 


\begin{tabular}{|c|c|c|}
\hline$P(x)$ & $m(P)$ & $m_{2}(P)$ \\
\hline$x^{8}+x^{5}-x^{4}+x^{3}+1$ & 0.2473585132 & 1.0980813745 \\
\hline$x^{10}+x^{9}-x^{7}-x^{6}-x^{5}-x^{4}-x^{3}+x+1$ & 0.1623576120 & 1.7447964556 \\
\hline$x^{10}-x^{6}+x^{5}-x^{4}+1$ & 0.1958888214 & 1.2863292447 \\
\hline$x^{10}+x^{7}+x^{5}+x^{3}+1$ & 0.2073323581 & 1.2320444893 \\
\hline$x^{10}-x^{8}+x^{5}-x^{2}+1$ & 0.2320881973 & 1.1704950485 \\
\hline$x^{10}+x^{8}+x^{7}+x^{5}+x^{3}+x^{2}+1$ & 0.2368364616 & 1.1914083866 \\
\hline$x^{10}+x^{9}-x^{5}+x+1$ & 0.2496548880 & 1.0309287773 \\
\hline$x^{12}+x^{11}+x^{10}-x^{8}-x^{7}-x^{6}-x^{5}-x^{4}+x^{2}+x+1$ & 0.2052121880 & 1.4738375004 \\
\hline$x^{12}+x^{11}+x^{10}+x^{9}-x^{6}+x^{3}+x^{2}+x+1$ & 0.2156970336 & 1.5143823478 \\
\hline$x^{12}+x^{11}-x^{7}-x^{6}-x^{5}+x+1$ & 0.2239804947 & 1.2059443050 \\
\hline$x^{12}+x^{10}+x^{7}-x^{6}+x^{5}+x^{2}+1$ & 0.2345928411 & 1.2434560052 \\
\hline$x^{12}+x^{10}+x^{9}+x^{8}+2 x^{7}+x^{6}+2 x^{5}+x^{4}+x^{3}+x^{2}+1$ & 0.2412336268 & 1.6324129051 \\
\hline$x^{14}+x^{11}-x^{10}-x^{7}-x^{4}+x^{3}+1$ & 0.1823436598 & 1.3885013172 \\
\hline$x^{14}-x^{12}+x^{7}-x^{2}+1$ & 0.1844998024 & 1.3845721865 \\
\hline$x^{14}-x^{12}+x^{11}-x^{9}+x^{7}-x^{5}+x^{3}-x^{2}+1$ & 0.2272100851 & 1.4763006621 \\
\hline$x^{14}+x^{11}+x^{10}+x^{9}+x^{8}+x^{7}+x^{6}+x^{5}+x^{4}+x^{3}+1$ & 0.2351686174 & 1.4352060397 \\
\hline$x^{14}+x^{13}-x^{8}-x^{7}-x^{6}+x+1$ & 0.2368858459 & 1.2498299096 \\
\hline$x^{14}+x^{13}+x^{12}-x^{9}-x^{8}-x^{7}-x^{6}-x^{5}+x^{2}+x+1$ & 0.2453300143 & 1.3362661982 \\
\hline$x^{14}+x^{13}-x^{11}-x^{7}-x^{3}+x+1$ & 0.2469561884 & 1.3898540050 \\
\hline
\end{tabular}

Analogously, we can translate the speculations about $L_{2}$ to the case of $L_{2 h}$ with $h>1$, a set that satisfies $L_{2 h} \subset\left[\left(\frac{\pi^{2}}{48}\right)^{h}, \infty\right)$.

On the other hand, we have proved that $L_{2 h+1}$ (for $h>0$ ) has positive and negative values. By taking powers, it is easy to build sequences of polynomials whose $m_{2 h+1}$ tend to either $\infty$ or $-\infty$. We have also seen that 0 is a limit point. Notice that this last fact is related to $m_{2 h+1}$ being nontrivial on cyclotomic polynomials, something that is not true in the case of the classical Mahler measure.

In conclusion, we see that $m_{k}(P)$ has very different behavior depending on the parity of $k$. We expect that $m_{k}(P)$ for $k>1$ is nontrivial for cyclotomic polynomials, and that this fact answers Lehmer's question for $k>1$.

Acknowledgements We would like to thank David Boyd for his feedback on this work and Kannan Soundararajan for his interest and his ideas on how to prove that sequences of values of high Mahler measures are not identically zero. Finally we would like to thank the referee for many helpful suggestions that have greatly improved the exposition of this note. 


\section{References}

1. R. Breusch, On the distribution of the roots of a polynomial with integral coefficients. Proc. Amer. Math. Soc. 2 (1951), 939-941.

2. D. W. Boyd, Speculations concerning the range of Mahler's measure. Canad. Math. Bull. 24 (1981), no. 4, 453-469.

3. D. W. Boyd, Kronecker's theorem and Lehmer's problem for polynomials in several variables. J. Number Theory 13 (1981), no. 1, 116-121.

4. C. Deninger, Deligne periods of mixed motives, $K$-theory and the entropy of certain $Z^{n}$-actions. J. Amer. Math. Soc. 10 (1997), no. 2, 259-281.

5. L. Kronecker, Zwei Sätze über Gleichungen mit ganzzahligen Coefficienten. J. Reine Angew. Math. 53 (1857), 173-175.

6. N. Kurokawa, M. Lalín and H. Ochiai, Higher Mahler measure and zeta functions. Acta Arith. 135 (2008), no. 3, 269-297.

7. M. Lalín, Higher Mahler measure as a Massey product in Deligne Cohomology. To appear in Low-Dimensional Topology and Number Theory. Abstracts from the workshop held August 15-21, 2010. Organized by Paul E. Gunnells, Walter Neumann, Adam S. Sikora, and Don Zagier. Oberwolfach Reports. Oberwolfach Rep. 2010.

8. W. Lawton, A problem of Boyd concerning geometric means of polynomials. J. Number Theory 16 (1983), no. 3, 356-362

9. D. H. Lehmer, Factorization of certain cyclotomic functions. Annals of Math. 2 vol. 34 (1933) 461-479.

10. K. Mahler, On two extremum properties of polynomials. Illinois J. Math. 7 (1963) 681-701.

11. M. Mossinghoff, Lehmer's Problem, Polynomial Searches (by Gavin Taylor) http://www.cecm.sfu.ca/ mjm/Lehmer/search/.

12. C. J. Smyth, On the product of the conjugates outside the unit circle of an algebraic integer. Bull. Lond. Math. Soc. 3 (1971), 169-175.

13. C. J. Smyth, The Mahler measure of algebraic numbers: a survey. Number theory and polynomials, 322-349, London Math. Soc. Lecture Note Ser., 352, Cambridge Univ. Press, Cambridge, 2008. 